\title{
Radu-Miheț Method for the Existence, Uniqueness, and Approximation of the $\psi$-Hilfer Fractional Equations by Matrix-Valued Fuzzy Controllers
}

\author{
Zahra Eidinejad ${ }^{1}$, Reza Saadati ${ }^{1, *(\mathbb{D})}$ and Manuel de la Sen ${ }^{2}(\mathbb{D}$ \\ 1 School of Mathematics, Iran University of Science and Technology, Narmak, Tehran 13114-16846, Iran; \\ zahra_eidinejad@mathdep.iust.ac.ir \\ 2 Campus of Leioa, Institute of Research and Development of Processes IIDP, University of the Basque Country, \\ 48940 Leioa, Spain; manuel.delasen@ehu.eus \\ * Correspondence: rsaadati@eml.cc or rsaadati@iust.ac.ir
}

Citation: Eidinejad, Z.; Saadati, R.; de la Sen, M. Radu-Miheț Method for the Existence, Uniqueness, and Approximation of the $\psi$-Hilfer Fractional Equations by Matrix-Valued Fuzzy Controllers. Axioms 2021, 10, 63. https://doi.org/ $10.3390 /$ axioms 10020063

Academic Editor: Wei-Shih Du

Received: 5 March 2021

Accepted: 14 April 2021

Published: 16 April 2021

Publisher's Note: MDPI stays neutral with regard to jurisdictional claims in published maps and institutional affiliations.

Copyright: (C) 2021 by the authors. Licensee MDPI, Basel, Switzerland. This article is an open access article distributed under the terms and conditions of the Creative Commons Attribution (CC BY) license (https:/ / creativecommons.org/licenses/by/ $4.0 /)$.

\begin{abstract}
We apply the Radu-Miheț method derived from an alternative fixed-point theorem with a class of matrix-valued fuzzy controllers to approximate a fractional Volterra integro-differential equation with the $\psi$-Hilfer fractional derivative in matrix-valued fuzzy $k$-normed spaces to obtain an approximation for this type of fractional equation.
\end{abstract}

Keywords: $\psi$-Hilfer fractional equation; Volterra integro-differential equation; MVF-k-N-spaces; approximation; Radu-Miheţ method

MSC: 46L05; 47B47; 47H10; 46L57; 39B62

\section{Introduction}

Fractional calculus is considered as a branch of mathematical analysis that deals with the investigation and applications of integrals and derivatives of arbitrary order. Therefore, fractional calculus is an extension of the integer-order calculus that considers integrals and derivatives of any real or complex order [1], i.e., unifying and generalizing the notions of integer-order differentiation and $n$-fold integration. Various forms of fractional operators have been introduced over time, such as the Riemann-Liouville, Grünwald-Letnikov, Weyl, Caputo, Marchaud, or Hadamard fractional derivatives. The first approach is the Riemann-Liouville one, which is based on the iteration of the classical integral operator for $n$ times and then considering Cauchy's formula where $n$ ! is replaced by the Gamma function; hence, the fractional integral of non-integer order is defined. Results on the existence and stability of solutions of implicit fractional differential equations can be found in [2-4]. In this article, we study the fuzzy sets' matrix valued with the generalized t-norms, to define a matrix-valued fuzzy $k$-Banach space (in short MV-k-FB-space) and introduce a new class of matrix-valued fuzzy controllers. We apply the Radu-Miheț method to get an approximation for a class of $\psi$-Hilfer fractional Volterra integro-differential equations [5] in the matrix-valued fuzzy $k$-normed spaces (MVF-k-N-spaces).

The paper is organized as follows. In the next section, we present the definition of the generalized t-norm and define the matrix-valued fuzzy $k$-normed space. Next, we introduce the matrix-valued fuzzy controllers and the concept of Hyers-Ulam-Rassias stability. In Section 3, we apply the Radu-Miheţ method derived from the alternative fixed point theorem to study the Hyers-Ulam-Rassias stability of fractional Volterra integrodifferential equations in MVF-k-B-spaces. In Section 4, we investigate the Hyers-UlamRassias stability of fractional Volterra integral equations in MVF-k-B-spaces. In Section 5, we present some examples to illustrate our main results. 


\section{Preliminaries}

Here, we let $E_{1}=[0, p], E_{2}=(0, \infty), E_{3}=(0,1], E_{4}=[0, \infty], E_{5}=[0,1]$ (note that $E_{5}^{\circ}=(0,1)$ denotes the interior of $\left.E_{5}\right)$, and $E_{6}=[0, \infty)$.

Let:

$$
\operatorname{diag} M_{n}\left(E_{5}\right)=\left\{\left[\begin{array}{lll}
q_{1} & & \\
& \ddots & \\
& & q_{n}
\end{array}\right]=\operatorname{diag}\left[q_{1}, \cdots, q_{n}\right], q_{1}, \ldots, q_{n} \in E_{5}\right\},
$$

where $\operatorname{diag} M_{n}\left(E_{5}\right)$ is equipped with the partial order relation:

$$
\begin{gathered}
\boldsymbol{q}=\operatorname{diag}\left[q_{1}, \cdots, q_{n}\right], \boldsymbol{b}=\operatorname{diag}\left[b_{1}, \cdots, b_{n}\right] \in \operatorname{diag} M_{n}\left(E_{5}\right), \\
\boldsymbol{q} \preceq \boldsymbol{b} \Longleftrightarrow q_{i} \leq b_{i} \text { for every } i=1, \ldots, n .
\end{gathered}
$$

Furthermore, $\boldsymbol{q} \prec \boldsymbol{b}$ denotes that $\boldsymbol{q} \preceq \boldsymbol{b}$ and $\boldsymbol{q} \neq \boldsymbol{b}$; $\boldsymbol{q} \ll \boldsymbol{b}$ and $a_{i}<b_{i}$ for every $i=1, \ldots, n$. We define $\boldsymbol{e}=\operatorname{diag}[e, \ldots, e]$ in $\operatorname{diag} M_{n}\left(E_{5}\right)$ where $e \in E_{5}$. For instance, $\mathbf{1}=$ $\operatorname{diag}[1, \ldots, 1]$ and $\mathbf{0}=\operatorname{diag}[0, \ldots, 0]$.

Now, we define a class of t-norms $[6,7]$ on $\operatorname{diag} M_{n}\left(E_{5}\right)$.

Definition 1. ([6-8]) Consider the generalized t-norm $(G T N) \circledast: \operatorname{diag} M_{n}\left(E_{5}\right) \times \operatorname{diag} M_{n}\left(E_{5}\right) \rightarrow$ $\operatorname{diag} M_{n}\left(E_{5}\right)$, which satisfies the following conditions:

(a) $\left.\left(\forall \boldsymbol{q} \in \operatorname{diag} M_{n}\left(E_{5}\right)\right)(\boldsymbol{q} \circledast \mathbf{1})=\boldsymbol{q}\right)$ (boundary condition);

(b) $\quad\left(\forall(\boldsymbol{q}, \boldsymbol{b}) \in\left(\operatorname{diag} M_{n}\left(E_{5}\right)\right)^{2}\right)(\boldsymbol{q} \circledast \boldsymbol{b}=\boldsymbol{b} \circledast \boldsymbol{q})$ (commutativity);

(c) $\quad\left(\forall(\boldsymbol{q}, \boldsymbol{b}, \boldsymbol{c}) \in\left(\operatorname{diag} M_{n}\left(E_{5}\right)\right)^{3}\right)(\boldsymbol{q} \circledast(\boldsymbol{b} \circledast \boldsymbol{c})=(\boldsymbol{q} \circledast \boldsymbol{b}) \circledast \boldsymbol{c})$ (associativity);

(d) $\quad\left(\forall\left(\boldsymbol{q}_{1}, \boldsymbol{q}_{2}, \boldsymbol{b}_{1}, \boldsymbol{b}_{2}\right) \in\left(\operatorname{diag} M_{n}\left(E_{5}\right)\right)^{4}\right)\left(\boldsymbol{q}_{1} \preceq \boldsymbol{q}_{2}\right.$ and $\left.\boldsymbol{b}_{1} \preceq \boldsymbol{b}_{2} \Longrightarrow \boldsymbol{q}_{1} \circledast \boldsymbol{b}_{1} \preceq \boldsymbol{q}_{2} \circledast \boldsymbol{b}_{2}\right)$ (monotonicity).

For every $\boldsymbol{q}, \boldsymbol{b} \in \operatorname{diag} M_{n}\left(E_{5}\right)$ and every sequences $\left\{\boldsymbol{q}_{k}\right\}$ and $\left\{\boldsymbol{b}_{k}\right\}$ converging to $\boldsymbol{q}$ and $\boldsymbol{b}$, suppose we have:

$$
\lim _{k}\left(\boldsymbol{q}_{k} \circledast \boldsymbol{b}_{k}\right)=\boldsymbol{q} \circledast \boldsymbol{b},
$$

then $\circledast$ on $\operatorname{diag} M_{n}\left(E_{5}\right)$ is the continuous GTN (CGTN). Now, we present some examples of the CGTN.

(1) Define $\circledast_{M}: \operatorname{diag} M_{n}\left(E_{5}\right) \times \operatorname{diag} M_{n}\left(E_{5}\right) \rightarrow \operatorname{diag} M_{n}\left(E_{5}\right)$, such that,

$$
\boldsymbol{q} \circledast_{M} \boldsymbol{b}=\operatorname{diag}\left[q_{1}, \cdots, q_{n}\right] \circledast_{M} \operatorname{diag}\left[b_{1}, \cdots, b_{n}\right]=\operatorname{diag}\left[\min \left\{q_{1}, b_{1}\right\}, \cdots, \min \left\{q_{n}, b_{n}\right\}\right],
$$

then $\circledast_{M}$ is the CGTN (minimum CGTN);

(2) Define $\circledast_{P}: \operatorname{diag} M_{n}\left(E_{5}\right) \times \operatorname{diag} M_{n}\left(E_{5}\right) \rightarrow \operatorname{diag} M_{n}\left(E_{5}\right)$, such that,

$$
\boldsymbol{q} \circledast{ }_{P} \boldsymbol{b}=\operatorname{diag}\left[q_{1}, \cdots, q_{n}\right] \circledast{ }_{P} \operatorname{diag}\left[b_{1}, \cdots, b_{n}\right]=\operatorname{diag}\left[q_{1} . b_{1}, \cdots, q_{n} . b_{n}\right],
$$

then $\circledast_{P}$ is the CGTN (product CGTN);

(3) Define $\circledast_{L}: \operatorname{diag} M_{n}\left(E_{5}\right) \times \operatorname{diag} M_{n}\left(E_{5}\right) \rightarrow \operatorname{diag} M_{n}\left(E_{5}\right)$, such that,

$\boldsymbol{q} \circledast{ }_{L} \boldsymbol{b}=\operatorname{diag}\left[q_{1}, \cdots, q_{n}\right] \circledast_{L} \operatorname{diag}\left[b_{1}, \cdots, b_{n}\right]=\operatorname{diag}\left[\max \left\{q_{1}+b_{1}-1,0\right\}, \cdots, \max \left\{q_{n}+b_{n}-1,0\right\}\right]$, then $\circledast_{P}$ is the CGTN (Lukasiewicz CGTN). 
Now, we present some numerical examples:

$$
\begin{aligned}
& \operatorname{diag}\left[\frac{1}{2}, \frac{3}{4}, 1, \frac{2}{3}, \frac{1}{4}\right] \circledast_{M} \operatorname{diag}\left[0, \frac{1}{3}, \frac{2}{5}, \frac{6}{7}, 1\right]=
\end{aligned}
$$

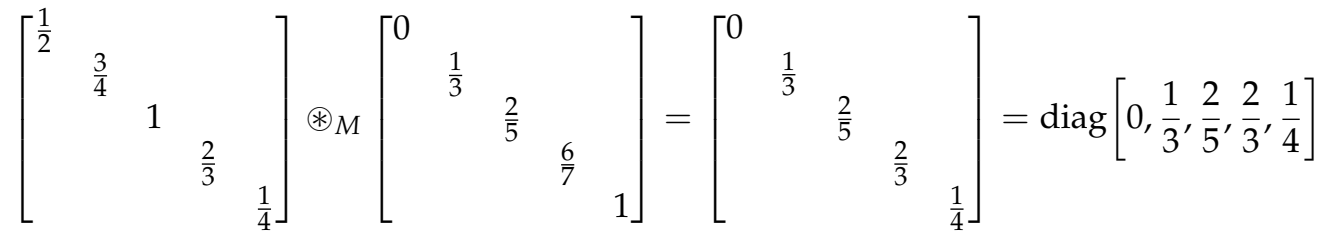

$$
\begin{aligned}
& \operatorname{diag}\left[\frac{1}{2}, \frac{3}{4}, 1, \frac{2}{3}, \frac{1}{4}\right] \circledast_{P} \operatorname{diag}\left[0, \frac{1}{3}, \frac{2}{5}, \frac{6}{7}, 1\right]=
\end{aligned}
$$

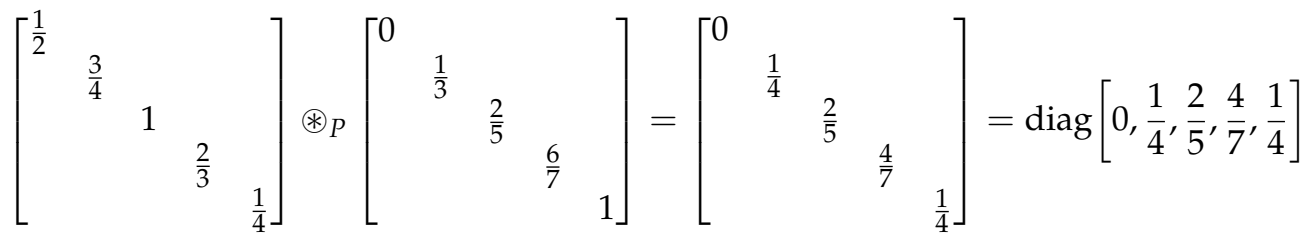

$$
\begin{aligned}
& \operatorname{diag}\left[\frac{1}{2}, \frac{3}{4}, 1, \frac{2}{3}, \frac{1}{4}\right] \circledast_{L} \operatorname{diag}\left[0, \frac{1}{3}, \frac{2}{5}, \frac{6}{7}, 1\right]=
\end{aligned}
$$

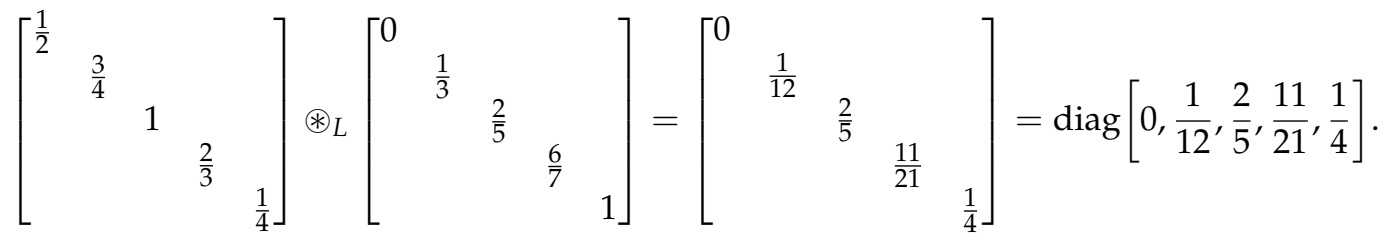

We get:

$$
\begin{aligned}
& \operatorname{diag}\left[\frac{1}{2}, \frac{3}{4}, 1, \frac{2}{3}, \frac{1}{4}\right] \circledast_{M} \operatorname{diag}\left[0, \frac{1}{3}, \frac{2}{5}, \frac{6}{7}, 1\right] \\
\succeq & \operatorname{diag}\left[\frac{1}{2}, \frac{3}{4}, 1, \frac{2}{3}, \frac{1}{4}\right] \circledast_{P} \operatorname{diag}\left[0, \frac{1}{3}, \frac{2}{5}, \frac{6}{7}, 1\right] \\
\succeq & \operatorname{diag}\left[\frac{1}{2}, \frac{3}{4}, 1, \frac{2}{3}, \frac{1}{4}\right] \circledast_{L} \operatorname{diag}\left[0, \frac{1}{3}, \frac{2}{5}, \frac{6}{7}, 1\right] .
\end{aligned}
$$

We consider the set of matrix-valued fuzzy functions (MVFFs) $\Phi$, which are left continuous and increasing functions $\varphi:\left(E_{1}\right)^{k} \times\left(E_{2}\right)^{n} \rightarrow \operatorname{diag} M_{n}\left(E_{3}\right)$, where $\vec{t}=\left(t_{1}, \cdots, t_{n}\right) \in$ $\left(E_{2}\right)^{n}$. Furthermore, $\lim _{t \rightarrow+\infty} \varphi\left(p_{1}, \cdots, p_{k}, \vec{t}\right)=\mathbf{1}$ for any $p_{1}, \cdots, p_{k}$ in $E_{1}$.

As an example, the matrix-valued fuzzy function $\varphi:\left(E_{1}\right)^{k} \times\left(E_{2}\right)^{3} \rightarrow \operatorname{diag} M_{n}\left(E_{3}\right)$ :

$\varphi\left(p_{1}, \cdots, p_{k}, \vec{t}\right)=\operatorname{diag}\left[\exp \left(\frac{-\left\|p_{1} \cdots, p_{k}\right\|}{t_{1}}\right), \frac{t_{2}}{t_{2}+\left\|p_{1}, \cdots, p_{k}\right\|}, \exp \left(\frac{-\left\|p_{1}, \cdots, p_{k}\right\|}{t_{3}}\right)\right]$,

for $\vec{t} \in\left(E_{2}\right)^{3}$.

In $\Phi$, we define “ $\preceq "$ as follows:

$\varphi \precsim \phi \Longleftrightarrow \varphi\left(p_{1}, \cdots, p_{k}, \vec{t}\right) \preceq \phi\left(p_{1}, \cdots, p_{k}, \vec{t}\right), \quad \forall \vec{t} \in\left(E_{2}\right)^{n}$ and $p_{1}, \cdots, p_{k} \in E_{1}$.

Definition 2. Consider the CGTN $\circledast$, a vector space $V$, and the matrix-valued fuzzy set (MVFS) $\Theta: V^{k} \times\left(E_{2}\right)^{n} \rightarrow \operatorname{diag} M_{n}\left(E_{3}\right)$. In this case, we define a matrix-valued fuzzy $k$-normed space (MVF-K-N-space) $(V, \Theta, \circledast)$ as:

$(M V F-K-N 1) \Theta\left(v_{1}, \cdots, v_{k}, \vec{t}\right)=1$ if and only if $v_{1}, \cdots, v_{k}$ are linearly dependent and $\vec{t} \in\left(E_{2}\right)^{n}$ 
$(M V F-K-N 2) \Theta\left(\alpha v_{1}, \cdots, v_{k}, \vec{t}\right)=\Theta\left(v_{1}, \cdots, v_{k}, \frac{\vec{t}}{|\alpha|}\right)$ for all $v_{1}, \cdots, v_{k} \in V$ and $\alpha \in \mathbb{C}$ with $\alpha \neq 0$;

$(M V F-K-N 3) \Theta\left(v_{0}+v_{1}, v_{2}, \cdots, v_{k}, \vec{t}+\vec{s}\right) \succeq \Theta\left(v_{0}, v_{2}, \cdots, v_{k}, \vec{t}\right) \circledast \Theta\left(v_{1}, v_{2}, \cdots, v_{k}, \vec{s}\right)$ for all $v_{1}, \cdots, v_{k} \in V$ and any $\vec{t} \in\left(E_{2}\right)^{n}$ and $\vec{s} \in\left(E_{2}\right)^{n}$;

$(M V F-K-N 4) \lim _{t \rightarrow+\infty} \Theta\left(p_{1}, \cdots, p_{k}, \vec{t}\right)=\mathbf{1}$ for any $\vec{t} \in\left(E_{2}\right)^{n}$.

A complete MVF-k-N-space is called a matrix-valued fuzzy Banach space (MVF-k-Bspace).

As an example, the matrix-valued fuzzy $k$-norm (MVF-k-N) $\Theta$,

$$
\begin{aligned}
& \Theta\left(v_{1}, v_{2}, \cdots, v_{k}, \vec{t}\right) \\
= & \operatorname{diag}\left[\exp \left(-\frac{\left\|v_{1}, v_{2}, \cdots, v_{k}\right\|}{t_{1}}\right), \frac{t_{2}}{t_{2}+\left\|v_{1}, v_{2}, \cdots, v_{k}\right\|}, \exp \left(-\frac{\left\|v_{1}, v_{2}, \cdots, v_{k}\right\|}{t_{3}}\right)\right],
\end{aligned}
$$

for $\vec{t} \in\left(E_{2}\right)^{3}$.

Define a matrix-valued fuzzy $k$-norm, and $\left(V, \Theta, \circledast_{M}\right)$ is an MVF-k-N-space; here, $(V,\|\cdot\|)$ is a $k$-normed vector space. In this paper, we assume that $\circledast=\circledast_{M}$.

Theorem 1 ([9,10]). Let $(W, d)$ be a complete $E_{4}$-valued metric space, and let $\Lambda: W \rightarrow W$ be a strictly contractive function with Lipschitz constant $\mathrm{\epsilon}<1$. Thus, for a given element $\rho \in W$, either:

$$
d\left(\Lambda^{n} \rho, \Lambda^{n+1} \rho\right)=\infty
$$

for each $n \in \mathbb{N}$ or there is $n_{0} \in \mathbb{N}$ such that:

(i) $d\left(\Lambda^{n} \rho, \Lambda^{n+1} \rho\right)<\infty$ for every $n \geq n_{0}$;

(ii) the fixed point $Q^{*}$ of $\Lambda$ is the convergent point of sequence $\left\{\Lambda^{n} \rho\right\}$;

(iii) in the set $U=\left\{\varrho \in W \mid d\left(\Lambda^{n_{0}} \rho, \varrho\right)<\infty\right\}$, $\varrho^{*}$ is the unique fixed point of $\Lambda$;

(iv) $(1-€) d\left(\varrho, \varrho^{*}\right) \leq d(\varrho, \Lambda \varrho)$ for every $\varrho \in U$.

Definition $3([5])$. Let $\Omega:(a, b) \rightarrow \mathbb{R}(0<a<b<\infty)$ be a finite interval and $\kappa>$ 0 . Furthermore, let $\psi(p)$ be an increasing and positive monotone function on $(a, b]$, having $a$ continuous derivative $\psi^{\prime}(p)$ (we denote the first derivative as $\frac{d}{d t} \psi(p)=\psi^{\prime}(p)$ on $(a, b)$ ). The leftsided fractional integral of a function $f$ with respect to a function $\psi(p)$ on $(a, b)$ is defined by:

$$
\mathcal{I}_{a^{+}}^{\kappa, \psi} \Omega(p)=\frac{1}{\Gamma(\kappa)} \int_{a}^{p} \mathcal{A}_{\psi}^{\kappa}(p, s) \Omega(s) d s, a<p,
$$

where $\mathcal{A}_{\psi}^{\kappa}(p, s):=\psi^{\prime}(p)(\psi(p)-\psi(s))^{\kappa-1}$. The right-sided fractional integral is defined in an analogous form.

As the aim of this paper is to present some types of stabilities involving a class of fractional integro-differential equations by means of a $\psi$-Hilfer fractional operator, we introduce such a fractional operator.

Definition 4. [5] Let $\kappa \in{\stackrel{\circ}{E_{5}}}, \Omega$ be an integrable function on $E_{1}$ and $\psi \in C^{1}\left(E_{1}\right)$ be an increasing function with $\psi^{\prime}(p) \neq 0$, for each $p \in E_{1}$. Define the $\psi$-Hilfer fractional derivative as:

$$
{ }^{H} \mathcal{D}_{0+}^{\kappa, \tau ; \psi} \Omega(p)=\mathcal{I}_{0+}^{\tau(1-\kappa) ; \psi}\left(\frac{1}{\psi^{\prime}(p)} \frac{d}{d p}\right) \mathcal{I}_{0+}^{(1-\tau)(1-\kappa) ; \psi} \Omega(p) .
$$

Consider the $\psi$-Hilfer fractional Volterra integro-differential equation, defined by:

$$
{ }^{H} \mathcal{D}_{0^{+}}^{\kappa, \tau, \psi} \Omega(p)=S(p, \omega(p))+\int_{0}^{p} \mathcal{M}(p, \sigma, \Omega(\sigma)) d \sigma,
$$


where $S(p, \omega(p))$ is a continuous function (CF) with respect to the variables $p, \omega$, and also, $\mathcal{M}(p, \sigma, \Omega(\sigma))$ is a CF with respect to $p, \sigma$ and $\Omega$ on $E_{1}, \kappa \in E_{5}^{\circ}, \tau \in E_{5}, S: E_{1} \times V \rightarrow V$, and $\mathcal{M}: E_{1} \times E_{1} \times V \rightarrow V$.

Let function $\phi:\left(E_{1}\right)^{k} \times\left(E_{2}\right)^{n} \rightarrow \operatorname{diag} M_{n}\left(E_{3}\right)$ be a matrix-valued fuzzy function. Equation (3) is said to be Hyers-Ulam-Rassias stable if $\Omega\left(p_{1}\right), \cdots, \Omega\left(p_{k}\right)$ is a given differentiable function, satisfying:

$$
\begin{aligned}
& \Theta\left({ }_{0} \mathcal{D}_{p_{1}}^{\kappa, \tau, \psi} \Omega\left(p_{1}\right)-S\left(p_{1}, \Omega\left(p_{1}\right)\right)-\int_{0}^{p_{1}} \mathcal{M}\left(p_{1}, \sigma_{1}, \Omega\left(\sigma_{1}\right)\right) d \sigma_{1}, \cdots,\right. \\
& \left.{ }_{0} \mathcal{D}_{p_{k}}^{\kappa, \tau, \psi} \Omega\left(p_{k}\right)-S\left(p_{k}, \Omega\left(p_{k}\right)\right)-\int_{0}^{p_{k}} \mathcal{M}\left(p_{k}, \sigma_{k}, \Omega\left(\sigma_{k}\right)\right) d \sigma_{k}, \vec{\mu}\right) \succeq \phi\left(p_{1}, \cdots, p_{k}, \vec{\mu}\right),
\end{aligned}
$$

for $p_{1}, \cdots, p_{k} \in E_{1}$, and we can find a solution $\mathrm{Y}\left(p_{1}\right) \cdots, \mathrm{Y}\left(p_{k}\right)$ of Equation (3) such that for some $r>0$,

$$
\Theta\left(\Omega\left(p_{1}\right)-\mathrm{Y}\left(p_{1}\right), \cdots, \Omega\left(p_{k}\right)-\mathrm{Y}\left(p_{k}\right), \vec{\mu}\right) \succeq \phi\left(p_{1}, \cdots, p_{k}, \frac{\vec{\mu}}{r}\right) .
$$

Using the Radu-Miheț method, we study the Hyers-Ulam-Rassias stability of the $\psi$-Hilfer fractional Volterra integro-differential equation (3) in MVF-k-B-space $(V, \Theta, \circledast)$. Our results can be applied to improve recent results [5], and by the methods used in this paper, we can extend some fractional Volterra integro-differential equations in MVF-k-Bspaces [11-14].

\section{Best Approximation $\psi$-Hilfer Fractional Volterra Integro-Differential Equation}

In this section, we apply the Radu-Miheț method derived from Theorem 1 to study the Hyers-Ulam-Rassias stability of functional Equation (3); for more details, we refer to $[15,16]$. Consider the MVF-k-B-space $(V, \Theta, \circledast)$ and matrix-valued fuzzy function (MVFF) $\phi:\left(E_{1}\right)^{k} \times\left(E_{2}\right)^{n} \rightarrow \operatorname{diag} M_{n}\left(E_{3}\right)$. We set:

$$
B:=\left\{\Omega: E_{1} \rightarrow V, \Omega \text { is differentiable }\right\}
$$

and define a mapping $d$ from $B \times B$ to $E_{4}$ by:

$$
\begin{gathered}
d(\Omega, Y)=\inf \left\{C \in E_{6}: \Theta\left({ }_{0} \mathcal{D}_{p_{1}}^{\kappa, \tau, \psi} \Omega\left(p_{1}\right)-{ }_{0} \mathcal{D}_{p_{1}}^{\kappa, \tau, \psi} Y\left(p_{1}\right), \cdots,{ }_{0} \mathcal{D}_{p_{k}}^{\kappa, \tau, \psi} \Omega\left(p_{k}\right)-{ }_{0} \mathcal{D}_{p_{k}}^{\kappa, \tau, \psi} Y\left(p_{k}\right), \vec{\mu}\right) \circledast\right. \\
\Theta\left(\Omega\left(p_{1}\right)-Y\left(p_{1}\right), \cdots, \Omega\left(p_{k}\right)-Y\left(p_{k}\right), \vec{\mu}\right), \succeq \phi\left(p_{1}, \cdots, p_{k}, \frac{\vec{\mu}}{C}\right), \\
\left.\forall \Omega, Y \in B, p_{1}, \cdots, p_{k} \in E_{1}, \vec{\mu} \in\left(E_{2}\right)^{n}\right\} .
\end{gathered}
$$

Theorem 2. $(B, d)$ is a complete $E_{4}$-valued metric fuzzy space.

Proof. First, we show that $(B, d)$ is an $E_{4}$-valued metric fuzzy space.

We show that $d(\Omega, \mathrm{Y})=0$ if and only if $\Omega=\mathrm{Y}$. Let $d(\Omega, \mathrm{Y})=0$; we have:

$$
\begin{aligned}
\inf \left\{C \in E_{6}: \Theta\left({ }_{0} \mathcal{D}_{p_{1}}^{\kappa, \tau, \psi} \Omega\left(p_{1}\right)-{ }_{0} \mathcal{D}_{p_{1}}^{\kappa, \tau, \psi} \mathrm{Y}\left(p_{1}\right), \cdots,{ }_{0} \mathcal{D}_{p_{k}}^{\kappa, \tau, \psi} \Omega\left(p_{k}\right)-{ }_{0} \mathcal{D}_{p_{k}}^{\kappa, \tau, \psi} \mathrm{Y}\left(p_{k}\right), \vec{\mu}\right) \circledast\right. \\
\Theta\left(\Omega\left(p_{1}\right)-\mathrm{Y}\left(p_{1}\right), \cdots, \Omega\left(p_{k}\right)-\mathrm{Y}\left(p_{k}\right), \vec{\mu}\right) \succeq \phi\left(p_{1}, \cdots, p_{k}, \frac{\vec{\mu}}{C}\right), \\
\left.\forall \Omega, Y \in B, p_{1}, \cdots, p_{k} \in E_{1}, \vec{\mu} \in\left(E_{2}\right)^{n}\right\}=0
\end{aligned}
$$


and so:

$$
\begin{aligned}
& \Theta\left({ }_{0} \mathcal{D}_{p_{1}}^{\kappa, \tau, \psi} \Omega\left(p_{1}\right)-{ }_{0} \mathcal{D}_{p_{1}}^{\kappa, \tau, \psi} \mathrm{Y}\left(p_{1}\right), \cdots,{ }_{0} \mathcal{D}_{p_{k}}^{\kappa, \tau, \psi} \Omega\left(p_{k}\right)-{ }_{0} \mathcal{D}_{p_{k}}^{\kappa, \tau, \psi} \mathrm{Y}\left(p_{k}\right), \vec{\mu}\right) \circledast \\
& \Theta\left(\Omega\left(p_{1}\right)-\mathrm{Y}\left(p_{1}\right), \cdots, \Omega\left(p_{k}\right)-\mathrm{Y}\left(p_{k}\right), \vec{\mu}\right) \succeq \phi\left(p_{1}, \cdots, p_{k}, \frac{\vec{\mu}}{C}\right),
\end{aligned}
$$

for all $C \in E_{6}$. $C$ tends to zero in the above inequality, and we get:

$$
\Theta\left({ }_{0} \mathcal{D}_{p_{1}}^{\kappa, \tau, \psi} \Omega\left(p_{1}\right)-{ }_{0} \mathcal{D}_{p_{1}}^{\kappa, \tau, \psi} \mathrm{Y}\left(p_{1}\right), \cdots{ }_{0} \mathcal{D}_{p_{k}}^{\kappa, \tau, \psi} \Omega\left(p_{k}\right)-{ }_{0} \mathcal{D}_{p_{k}}^{\kappa, \tau, \psi} \mathrm{Y}\left(p_{k}\right), \vec{\mu}\right)=\mathbf{1}
$$

and so:

$$
\Theta\left(\Omega\left(p_{1}\right)-\mathrm{Y}\left(p_{1}\right), \cdots, \Omega\left(p_{k}\right)-\mathrm{Y}\left(p_{k}\right), \vec{\mu}\right)=\mathbf{1}
$$

Thus, $\Omega\left(p_{1}, \cdots, p_{k}\right)=\mathrm{Y}\left(p_{1}, \cdots, p_{k}\right)$ for every $p_{1}, \cdots, p_{k} \in E_{1}$, and vice versa. It is straight forward to show $d(\Omega, \mathrm{Y})=d(\Omega, \mathrm{Y})$ for every $\Omega, \mathrm{Y} \in B$. Now, let $d(\Omega, \mathrm{Y})=e_{1} \in E_{2}$ and $d(\Omega, \mathrm{Y})=e_{2} \in E_{2}$. Then, we have:

$$
\begin{aligned}
& \Theta\left({ }_{0} \mathcal{D}_{p_{1}}^{\kappa, \tau, \psi} \Omega\left(p_{1}\right)-{ }_{0} \mathcal{D}_{p_{1}}^{\kappa, \tau, \psi} \mathrm{Y}\left(p_{1}\right), \cdots,{ }_{0} \mathcal{D}_{p_{k}}^{\kappa, \tau, \psi} \Omega\left(p_{k}\right)-{ }_{0} \mathcal{D}_{p_{k}}^{\kappa, \tau, \psi} \mathrm{Y}\left(p_{k}\right), \vec{\mu}\right) \circledast \\
& \Theta\left(\Omega\left(p_{1}\right)-\mathrm{Y}\left(p_{1}\right), \cdots, \Omega\left(p_{k}\right)-\mathrm{Y}\left(p_{k}\right), \vec{\mu}\right) \succeq \phi\left(p_{1}, \cdots, p_{k}, \frac{\vec{\mu}}{e_{1}}\right),
\end{aligned}
$$

and:

$$
\begin{aligned}
& \Theta\left({ }_{0} \mathcal{D}_{p_{1}}^{\kappa, \tau, \psi} \mathrm{Y}\left(p_{1}\right)-{ }_{0} \mathcal{D}_{p_{1}}^{\kappa, \tau, \psi} \omega\left(p_{1}\right), \cdots,{ }_{0} \mathcal{D}_{p_{k}}^{\kappa, \tau, \psi} \mathrm{Y}\left(p_{k}\right)-{ }_{0} \mathcal{D}_{p_{k}}^{\kappa, \tau, \psi} \omega\left(p_{k}\right), \vec{\mu}\right) \circledast \\
& \Theta\left(\mathrm{Y}\left(p_{1}\right)-\omega\left(p_{1}\right), \cdots, \mathrm{Y}\left(p_{k}\right)-\omega\left(p_{k}\right), \vec{\mu}\right) \succeq \phi\left(p_{1}, \cdots, p_{k}, \frac{\vec{\mu}}{e_{2}}\right),
\end{aligned}
$$

for every $\vec{\mu} \in\left(E_{2}\right)^{n}$. Then, we have:

$$
\begin{aligned}
& \Theta\left({ }_{0} \mathcal{D}_{p_{1}}^{\kappa, \tau, \psi} \Omega\left(p_{1}\right)-{ }_{0} \mathcal{D}_{p_{1}}^{\kappa, \tau, \psi} \omega\left(p_{1}\right), \cdots{ }_{0} \mathcal{D}_{p_{k}}^{\kappa, \tau, \psi} \Omega\left(p_{k}\right)-{ }_{0} \mathcal{D}_{p_{k}}^{\kappa, \tau, \psi} \omega\left(p_{k}\right),\left(e_{1}+e_{2}\right) \vec{\mu}\right) \circledast \\
& \Theta\left(\Omega\left(p_{1}\right)-\omega\left(p_{1}\right), \cdots, \Omega\left(p_{k}\right)-\omega\left(p_{k}\right),\left(e_{1}+e_{2}\right) \vec{\mu}\right) \\
& \succeq\left[\Theta\left({ }_{0} \mathcal{D}_{p_{1}}^{\kappa, \tau, \psi} \Omega\left(p_{1}\right)-{ }_{0} \mathcal{D}_{p_{1}}^{\kappa, \tau, \psi} \mathrm{Y}\left(p_{1}\right), \cdots{ }_{0} \mathcal{D}_{p_{k}}^{\kappa, \tau, \psi} \Omega\left(p_{k}\right)-{ }_{0} \mathcal{D}_{p_{k}}^{\kappa, \tau, \psi} \mathrm{Y}\left(p_{k}\right), e_{1} \vec{\mu}\right) \circledast\right. \\
& \left.\Theta\left({ }_{0} \mathcal{D}_{p_{1}}^{\kappa, \tau, \psi} Y\left(p_{1}\right)-{ }_{0} \mathcal{D}_{p_{1}}^{\kappa, \tau} \omega\left(p_{1}\right), \cdots,{ }_{0} \mathcal{D}_{p_{k}}^{\kappa, \tau, \psi} Y\left(p_{k}\right)-{ }_{0} \mathcal{D}_{p_{k}}^{\kappa, \tau, \psi} \omega\left(p_{k}\right), e_{2} \vec{\mu}\right)\right] \\
& \circledast\left[\Theta\left(\Omega\left(p_{1}\right)-\mathrm{Y}\left(p_{1}\right), \cdots, \Omega\left(p_{k}\right)-\mathrm{Y}\left(p_{k}\right), e_{1} \vec{\mu}\right) \circledast \Theta\left(Y\left(p_{1}\right)-\omega\left(p_{1}\right), \cdots, \mathrm{Y}\left(p_{k}\right)-\omega\left(p_{k}\right), e_{2} \vec{\mu}\right)\right] \\
& \succeq \phi\left(p_{1}, \cdots, p_{k}, \vec{\mu}\right) \circledast \phi\left(p_{1}, \cdots, p_{k}, \vec{\mu}\right) \\
& =\phi\left(p_{1}, \cdots, p_{k}, \vec{\mu}\right)
\end{aligned}
$$

and so, $d(\Omega, \omega) \leq e_{1}+e_{2}$. Thus, $d(\Omega, \omega) \leq d(\Omega, \mathrm{Y})+d(\mathrm{Y}, \omega)$. Now, we are ready to prove $(B, d)$ is complete. Suppose that $\left\{\Omega_{k}\right\}_{k}$ is a Cauchy sequence in $(B, d)$. Let $p_{1}, \cdots, p_{k} \in E_{1}$. Assume that $\vec{v} \in\left(E_{2}\right)^{n}$ and $\lambda \in E_{5}{ }^{\circ}$ are arbitrary, and consider $\vec{\mu} \in\left(E_{2}\right)^{n}$ such that $\phi\left(p_{1}, \cdots, p_{k}, \vec{\mu}\right) \succ 1-\lambda$ for each $\lambda$ in $(0,1)$. For $e \vec{\mu}<\vec{v}$, choose $k_{0} \in \mathbb{N}$ such that:

$$
d\left(\Omega_{k}, \Omega_{\ell}\right)<e \quad \forall k, \ell \geq k_{0} .
$$


Then:

$$
\begin{aligned}
& \Theta\left({ }_{0} \mathcal{D}_{p_{1}}^{\kappa, \tau, \psi} \Omega_{k}\left(p_{1}\right)-{ }_{0} \mathcal{D}_{p_{1}}^{\kappa, \tau, \psi} \Omega_{l}\left(p_{1}\right), \cdots,{ }_{0} \mathcal{D}_{p_{k}}^{\kappa, \tau, \psi} \Omega_{k}\left(p_{k}\right)-{ }_{0} \mathcal{D}_{p_{k}}^{\kappa, \tau, \psi} \Omega_{l}\left(p_{k}\right), \vec{v}\right) \circledast \\
& \Theta\left(\Omega_{k}\left(p_{1}\right)-\Omega_{l}\left(p_{1}\right), \cdots, \Omega_{k}\left(p_{k}\right)-\Omega_{l}\left(p_{k}\right), \vec{v}\right) \succeq \\
& \Theta\left({ }_{0} \mathcal{D}_{p_{1}}^{\kappa, \tau} \Omega_{k}\left(p_{1}\right)-{ }_{0} \mathcal{D}_{p_{1}}^{\kappa, \tau} \Omega_{l}\left(p_{1}\right), \cdots,{ }_{0} \mathcal{D}_{p_{k}}^{\kappa, \tau, \psi} \Omega_{k}\left(p_{k}\right)-{ }_{0} \mathcal{D}_{p_{k}}^{\kappa, \tau, \psi} \Omega_{l}\left(p_{k}\right), e \vec{\mu}\right) \circledast \\
& \Theta\left(\Omega_{k}\left(p_{1}\right)-\Omega_{l}\left(p_{1}\right), \cdots, \Omega_{k}\left(p_{k}\right)-\Omega_{l}\left(p_{k}\right), e \vec{\mu}\right) \\
& \succeq \phi\left(p_{1}, \cdots, p_{k}, \vec{\mu}\right) \\
& \succ 1-\lambda .
\end{aligned}
$$

Then:

$$
\Theta\left({ }_{0} \mathcal{D}_{p_{1}}^{\kappa, \tau, \psi} \Omega_{k}\left(p_{1}\right)-{ }_{0} \mathcal{D}_{p_{1}}^{\kappa, \tau, \psi} \Omega_{l}\left(p_{1}\right), \cdots{ }_{0} \mathcal{D}_{p_{k}}^{\kappa, \tau, \psi} \Omega_{k}\left(p_{k}\right)-{ }_{0} \mathcal{D}_{p_{k}}^{\kappa, \tau, \psi} \Omega_{l}\left(p_{k}\right), \vec{v}\right) \succ 1-\lambda
$$

and $\Theta\left(\Omega_{k}\left(p_{1}\right)-\Omega_{l}\left(p_{1}\right), \cdots, \Omega_{k}\left(p_{k}\right)-\Omega_{l}\left(p_{k}\right), \vec{v}\right) \succ 1-\lambda$, i.e., the sequences $\left\{\Omega_{k}\left(p_{1}\right), \Omega_{k}\left(p_{2}\right), \cdots, \Omega_{k}\left(p_{k}\right)\right\}_{k}$ and $\left\{0 \mathcal{D}_{p_{1}}^{\kappa, \tau, \psi} \Omega_{k}\left(p_{1}\right){ }_{, 0} \mathcal{D}_{p_{2}}^{\kappa, \tau, \psi} \Omega_{k}\left(p_{2}\right), \cdots, 0 \mathcal{D}_{p_{k}}^{\kappa, \tau, \psi} \Omega_{k}\left(p_{k}\right)\right\}_{k}$ are Cauchy in complete space $(V, \Theta, \circledast)$ on compact set $E_{1}$, so they are uniformly convergent to the mapping $\Omega: E_{1} \rightarrow V$ and ${ }_{0} \mathcal{D}_{p_{1}}^{\kappa, \tau, \psi} \Omega,_{0} \mathcal{D}_{p_{2}}^{\kappa, \tau, \psi} \Omega, \cdots,{ }_{0} \mathcal{D}_{p_{k}}^{\kappa, \tau, \psi} \Omega$, respectively. The uniform convergence leads us to the fact that $\Omega$ is differentiable, i.e., an element of $B$; then, $(B, d)$ is complete.

Now, we are ready to study the Hyers-Ulam-Rassias stability of the $\psi$-Hilfer fractional Volterra integro-differential Equation (3) and get the best approximation with a better estimate for the $\psi$-Hilfer fractional Volterra integro-differential equation.

Theorem 3. Let $(V, \Theta, \circledast)$ be an MVFB-space and $L_{1}, L_{2}, L_{3}, L_{4}$ and $P$ be positive constant such that $N_{1}>2$ where $N_{1}=\min \left[L_{1}, L_{2} L_{3}, L_{1} L_{4}, L_{2} L_{3} L_{4}\right]$. Assume that the continuous mappings $S: E_{1} \times V \rightarrow V, \mathcal{M}: E_{1} \times E_{1} \times V \rightarrow V$ with matrix-valued fuzzy function $\phi:\left(E_{1}\right)^{k} \times\left(E_{2}\right)^{n} \rightarrow \operatorname{diag} M_{n}\left(E_{3}\right)$ satisfying:

$$
\begin{aligned}
& \Theta\left(S\left(p_{1}, \Omega\left(p_{1}\right)\right)-\boldsymbol{S}\left(p_{1}, \mathrm{Y}\left(p_{1}\right)\right), \cdots, \boldsymbol{S}\left(p_{k}, \Omega\left(p_{k}\right)\right)-\boldsymbol{S}\left(p_{k}, \mathrm{Y}\left(p_{k}\right)\right), \vec{\mu}\right) \succeq \\
& \Theta\left(\Omega\left(p_{1}\right)-\mathrm{Y}\left(p_{1}\right), \cdots, \Omega\left(p_{k}\right)-\mathrm{Y}\left(p_{k}\right), L_{1} \vec{\mu}\right), \\
& \Theta\left(\mathcal{M}\left(p_{1}, \sigma_{1}, \Omega\left(\sigma_{1}\right)\right)-\mathcal{M}\left(p_{1}, \sigma_{1}, \mathrm{Y}\left(\sigma_{1}\right)\right), \cdots, \mathcal{M}\left(p_{k}, \sigma_{k}, \Omega\left(\sigma_{k}\right)\right)-\mathcal{M}\left(p_{k}, \sigma_{k}, \mathrm{Y}\left(\sigma_{k}\right), \vec{\mu}\right) \succeq\right. \\
& \Theta\left(\Omega\left(\sigma_{1}\right)-\mathrm{Y}\left(\sigma_{1}\right), \cdots, \Omega\left(\sigma_{k}\right)-\mathrm{Y}\left(\sigma_{k}\right), L_{2} \vec{\mu}\right), \\
& \inf _{\xi_{1}, \cdots, \xi_{k} \in E_{1}} \phi\left(\xi_{1}, \cdots, \xi_{k}, \vec{\mu}\right) \succeq \phi\left(p_{1}, \cdots, p_{k}, L_{3} P \vec{\mu}\right),
\end{aligned}
$$

and:

$$
\begin{aligned}
& \Theta\left(\Omega\left(p_{1}\right), \Omega\left(p_{2}\right), \cdots, \Omega\left(p_{k}\right), \vec{\mu}\right) \succeq \phi\left(p_{1}, \cdots, p_{k}, \vec{\mu}\right), \text { implies that } \\
& \Theta\left({ }_{0} \mathcal{I}_{p_{1}}^{\kappa, \psi} \Omega\left(\sigma_{1}\right) d \sigma_{1}, \cdots,{ }_{0} \mathcal{I}_{p_{k}}^{\kappa, \psi} \Omega\left(\sigma_{k}\right) d \sigma_{K}, \vec{\mu}\right) \succeq \phi\left(p_{1}, \cdots, p_{k}, L_{4} \vec{\mu}\right),
\end{aligned}
$$

for every $p_{1}, \cdots, p_{k} \in E_{1}, \Omega, \mathrm{Y}: E_{1} \rightarrow V$, and $\vec{\mu} \in\left(E_{2}\right)^{n}$. 
Let $\omega: E_{1} \rightarrow V$ be a differentiable function satisfying:

$$
\begin{aligned}
& \Theta\left({ }_{0} \mathcal{D}_{p_{1}}^{\kappa, \tau, \psi} \omega\left(p_{1}\right)-S\left(p_{1}, \omega\left(p_{1}\right)\right)-\int_{0}^{p_{1}} \mathcal{M}\left(p_{1}, \sigma_{1}, \omega\left(\sigma_{1}\right)\right) d \sigma_{1}, \cdots,\right. \\
& \left.{ }_{0} \mathcal{D}_{p_{k}}^{\kappa, \tau, \psi} \omega\left(p_{k}\right)-S\left(p_{k}, \omega\left(p_{k}\right)\right)-\int_{0}^{p_{k}} \mathcal{M}\left(p_{k}, \sigma_{k}, \omega\left(\sigma_{k}\right)\right) d \sigma_{k}, \vec{\mu}\right) \\
\succeq & \phi\left(p_{1}, \cdots, p_{k}, \vec{\mu}\right),
\end{aligned}
$$

for every $p_{1}, \cdots, p_{k} \in E_{1}$ and $\vec{\mu} \in\left(E_{2}\right)^{n}$. Therefore, we are able find a unique differentiable function $\omega_{0}: E_{1} \rightarrow V$ such that:

$$
{ }_{0} \mathcal{D}_{p}^{\kappa, \tau, \psi} \omega_{0}(p)=\boldsymbol{S}\left(p, \omega_{0}(p)\right)+\int_{0}^{p} \mathcal{M}\left(p, \sigma, \omega_{0}(\sigma)\right) d \sigma
$$

and:

$$
\begin{aligned}
& \Theta\left({ }_{0} \mathcal{D}_{p_{1}}^{\kappa, \tau, \psi} \omega\left(p_{1}\right)-{ }_{0} \mathcal{D}_{p_{1}}^{\kappa, \tau, \psi} \omega_{0}\left(p_{1}\right), \cdots,{ }_{0} \mathcal{D}_{p_{k}}^{\kappa, \tau, \psi} \omega\left(p_{k}\right)-{ }_{0} \mathcal{D}_{p_{k}}^{\kappa, \tau, \psi} \omega_{0}\left(p_{k}\right), \vec{\mu}\right) \circledast \\
& \Theta\left(\omega\left(p_{1}\right)-\omega_{0}\left(p_{1}\right), \cdots, \omega\left(p_{k}\right)-\omega_{0}\left(p_{k}\right), \vec{\mu}\right) \succeq \\
& \phi\left(p_{1}, \cdots, p_{k}, \frac{N_{2}}{N_{3}} \vec{\mu}\right),
\end{aligned}
$$

for every $p_{1}, \cdots, p_{k} \in E_{1}$ and $\vec{\mu} \in\left(E_{2}\right)^{n}$, and $N_{2}=\min \left[1, L_{4}\right]$ and $N_{3}=\frac{\min \left[L_{1}, L_{2} L_{3}, L_{1} L_{4}, L_{2} L_{3} L_{4}\right]}{\min \left[L_{1}, L_{2} L_{3}, L_{1} L_{4}, L_{2} L_{3} L_{4}\right]-2}$.

Proof. We set:

$$
B:=\left\{\Omega: E_{1} \rightarrow V, \Omega \text { is differentiable }\right\}
$$

and introduce the $E_{4}$-valued metric on $B$ as,

$$
\begin{gathered}
d(\Omega, \mathrm{Y})=\inf \left\{C \in E_{6}: \Theta\left({ }_{0} \mathcal{D}_{p_{1}}^{\kappa, \tau, \psi} \Omega\left(p_{1}\right)-{ }_{0} \mathcal{D}_{p_{1}}^{\kappa, \tau, \psi} \mathrm{Y}\left(p_{1}\right), \cdots,{ }_{0} \mathcal{D}_{p_{k}}^{\kappa, \tau, \psi} \Omega\left(p_{k}\right)-{ }_{0} \mathcal{D}_{p_{k}}^{\kappa, \tau, \psi} \mathrm{Y}\left(p_{k}\right), \vec{\mu}\right)\right. \\
\circledast \Theta\left(\Omega\left(p_{1}\right)-\mathrm{Y}\left(p_{1}\right), \cdots, \Omega\left(p_{k}\right)-\mathrm{Y}\left(p_{k}\right), \vec{\mu}\right) \succeq \phi\left(p_{1}, \cdots, p_{k}, \frac{\vec{\mu}}{C}\right), \\
\left.\forall \Omega, \mathrm{Y} \in B, p_{1}, \cdots, p_{k} \in E_{1}, \vec{\mu} \in\left(E_{2}\right)^{n}\right\} .
\end{gathered}
$$

By Theorem 2, we have that $(B, d)$ is a complete $E_{4}$-valued metric space. Now, we define the mapping $\Lambda$ from $B$ to $B$ by:

$$
\Lambda\left(\Omega\left(p_{i}\right)\right)={ }_{0} \mathcal{I}_{p_{i}}^{\kappa, \psi}(\boldsymbol{S}(\sigma, \Omega(\sigma)))+{ }_{0} \mathcal{I}_{p_{i}}^{\kappa, \psi}\left(\int_{0}^{\sigma} \mathcal{M}(\sigma, \varepsilon, \Omega(\varepsilon)) d \varepsilon\right)
$$

where $\kappa \in E_{5}^{\circ}, S: E_{1} \times V \rightarrow V, \mathcal{M}: E_{1} \times E_{1} \times V \rightarrow V$, and $p_{i} \in E_{1}(i=1,2, \cdots, k)$. We prove $\Lambda$ is a strictly contractive mapping. Let $\Omega, Y \in B, C \in E_{2}$, and $d(\Omega, Y)<\vartheta$, then we have:

$$
\begin{aligned}
& \Theta\left({ }_{0} \mathcal{D}_{p_{1}}^{\kappa, \tau, \psi} \Omega\left(p_{1}\right)-{ }_{0} \mathcal{D}_{p_{1}}^{\kappa, \tau, \psi} \mathrm{Y}\left(p_{1}\right), \cdots{ }_{0} \mathcal{D}_{p_{k}}^{\kappa, \tau, \psi} \Omega\left(p_{k}\right)-{ }_{0} \mathcal{D}_{p_{k}}^{\kappa, \tau, \psi} \mathrm{Y}\left(p_{k}\right), \vartheta \vec{\mu}\right) \circledast \\
& \Theta\left(\Omega\left(p_{1}\right)-\mathrm{Y}\left(p_{1}\right), \vartheta \vec{\mu}\right) \succeq \phi\left(p_{1}, \cdots, p_{k}, \vec{\mu}\right), \\
& \forall \Omega, \mathrm{Y} \in B, p_{1}, \cdots, p_{k} \in E_{1}, \vec{\mu} \in\left(E_{2}\right)^{n} .
\end{aligned}
$$

Using the properties (MVF-K-N2) and (MVF-K-N3) of Definition 2 and (11), we have: 


$$
\begin{array}{ll} 
& \Theta\left({ }_{0} \mathcal{D}_{p_{1}}^{\kappa, \tau} \Lambda\left(\Omega\left(p_{1}\right)\right)-{ }_{0} \mathcal{D}_{p_{1}}^{\kappa, \tau, \psi} \Lambda\left(\mathrm{Y}\left(p_{1}\right)\right), \cdots,{ }_{0} \mathcal{D}_{p_{k}}^{\kappa, \tau, \psi} \Lambda\left(\Omega\left(p_{k}\right)\right)-{ }_{0} \mathcal{D}_{p_{k}}^{\kappa, \tau, \psi} \Lambda\left(\mathrm{Y}\left(p_{k}\right)\right), 2 \vartheta \vec{\mu}\right) \\
\circledast \quad & \Theta\left(\Lambda\left(\Omega\left(p_{1}\right)\right)-\Lambda\left(\mathrm{Y}\left(p_{1}\right)\right), \cdots, \Lambda\left(\Omega\left(p_{k}\right)\right)-\Lambda\left(\mathrm{Y}\left(p_{k}\right)\right), 2 \vartheta \vec{\mu}\right) \\
= & \Theta\left(\left[S\left(p_{1}, \Omega\left(p_{1}\right)\right)-S\left(p_{1}, \mathrm{Y}\left(p_{1}\right)\right)\right]+\int_{0}^{p_{1}}\left[\mathcal{M}\left(p_{1}, \sigma_{1}, \Omega\left(\sigma_{1}\right)\right)-\mathcal{M}\left(p_{1}, \sigma_{1}, \mathrm{Y}\left(\sigma_{1}\right)\right)\right] d \sigma_{1},\right. \\
\ldots, & \left.\left[S\left(p_{k}, \Omega\left(p_{k}\right)\right)-S\left(p_{k}, \mathrm{Y}\left(p_{k}\right)\right)\right]+\int_{0}^{p_{k}}\left[\mathcal{M}\left(p_{k}, \sigma_{k}, \Omega\left(\sigma_{k}\right)\right)-\mathcal{M}\left(p_{k}, \sigma_{k}, \mathrm{Y}\left(\sigma_{k}\right)\right)\right] d \sigma_{k}, 2 \vartheta \vec{\mu}\right) \\
\circledast \quad & \Theta\left({ }_{0} \mathcal{I}_{p_{1}}^{\kappa, \psi}\left(S\left(p_{1}, \Omega\left(p_{1}\right)\right)-S\left(p_{1}, \mathrm{Y}\left(p_{1}\right)\right)\right)+{ }_{0} \mathcal{I}_{p_{1}}^{\kappa, \psi}\left(\int_{0}^{p_{1}}\left[\mathcal{M}\left(p_{1}, \sigma_{1}, \Omega\left(\sigma_{1}\right)\right)-\mathcal{M}\left(p_{1}, \sigma_{1}, \mathrm{Y}\left(\sigma_{1}\right)\right)\right] d \sigma_{1}\right),\right. \\
\ldots, & { }_{0} \mathcal{I}_{p_{k}}^{\kappa, \psi}\left(S\left(p_{k}, \Omega\left(p_{k}\right)\right)-S\left(p_{k}, \mathrm{Y}\left(p_{k}\right)\right)\right)+ \\
& \left.{ }_{0} \mathcal{I}_{p_{k}}^{\kappa, \psi}\left(\int_{0}^{p_{k}}\left[\mathcal{M}\left(p_{k}, \sigma_{k}, \Omega\left(\sigma_{k}\right)\right)-\mathcal{M}\left(p_{k}, \sigma_{k}, \mathrm{Y}\left(\sigma_{k}\right)\right)\right] d \sigma_{k}\right), 2 \vartheta \vec{\mu}\right) \\
\succeq \quad & \Theta\left(S\left(p_{1}, \Omega\left(p_{1}\right)\right)-S\left(p_{1}, \mathrm{Y}\left(p_{1}\right)\right), \cdots, S\left(p_{k}, \Omega\left(p_{k}\right)\right)-S\left(p_{k}, \mathrm{Y}\left(p_{k}\right)\right), \vartheta \vec{\mu}\right) \\
\circledast \quad & \Theta\left(\int_{0}^{p_{1}}\left[\mathcal{M}\left(p_{1}, \sigma_{1}, \Omega\left(\sigma_{1}\right)\right)-\mathcal{M}\left(p_{1}, \sigma_{1}, \mathrm{Y}\left(\sigma_{1}\right)\right)\right] d \sigma_{1},\right. \\
\ldots, & \left.\int_{0}^{p_{k}}\left[\mathcal{M}\left(p_{k}, \sigma_{k}, \Omega\left(\sigma_{k}\right)\right)-\mathcal{M}\left(p_{k}, \sigma_{k}, \mathrm{Y}\left(\sigma_{k}\right)\right)\right] d \sigma_{k}, \vartheta \vec{\mu}\right) \\
\circledast \quad & \Theta\left({ }_{0} \mathcal{I}_{p_{1}}^{\kappa, \psi}\left[S\left(p_{1}, \Omega\left(p_{1}\right)\right)-S\left(p_{1}, \mathrm{Y}\left(p_{1}\right)\right)\right], \ldots{ }_{0} \mathcal{I}_{p_{k}}^{\kappa, \psi}\left[S\left(p_{k}, \Omega\left(p_{k}\right)\right)-S\left(p_{k}, \mathrm{Y}\left(p_{k}\right)\right)\right], \vartheta \vec{\mu}\right) \\
\circledast \quad & \Theta\left({ }_{0} \mathcal{I}_{p_{1}}^{\kappa, \psi} \int_{0}^{p_{1}}\left[\mathcal{M}\left(p_{1}, \sigma_{1}, \Omega\left(\sigma_{1}\right)\right)-\mathcal{M}\left(p_{1}, \sigma_{1}, Y\left(\sigma_{1}\right)\right)\right] d \sigma_{1},\right. \\
\ldots, & \left.{ }_{0} \mathcal{I}_{p_{k}}^{\kappa, \psi} \int_{0}^{p_{k}}\left[\mathcal{M}\left(p_{k}, \sigma_{k}, \Omega\left(\sigma_{k}\right)\right)-\mathcal{M}\left(p_{k}, \sigma_{k}, Y\left(\sigma_{k}\right)\right)\right] d \sigma_{k}, \vartheta \vec{\mu}\right) .
\end{array}
$$

In the last part of (12), there are four formulas, in the next steps, we work on them to get new formulas derived from the control function $\phi$. Let $0=\bar{y}_{1}<\bar{y}_{2}<\cdots<\bar{y}_{k}=p_{i}$, $\Delta \bar{y}_{i}=\bar{y}_{i}-\bar{y}_{i-1}=\frac{p_{i}}{k}, i=1,2, \cdots, k$, and $\|\Delta \bar{y}\|=\max _{1 \leq i \leq k}\left(\Delta \bar{y}_{i}\right)$.

Step 1. From (4), we have:

$$
\begin{aligned}
& \Theta\left(S\left(p_{1}, \Omega\left(p_{1}\right)\right)-S\left(p_{1}, Y\left(p_{1}\right)\right), \cdots, S\left(p_{k}, \Omega\left(p_{k}\right)\right)-S\left(p_{k}, Y\left(p_{k}\right)\right), \vartheta \vec{\mu}\right) \succeq \\
& \Theta\left(\Omega\left(p_{1}\right)-Y\left(p_{1}\right), \cdots, \Omega\left(p_{k}\right)-Y\left(p_{k}\right), L_{1} \vartheta \vec{\mu}\right) \succeq \phi\left(p_{1}, \cdots, p_{k}, L_{1} \vec{\mu}\right) .
\end{aligned}
$$

Step 2. Using (MVF-K-N2) and (MVF-K-N3) of Definition 2, the continuity property of MVFF $\Theta$, (5), and (6), we get:

$$
\begin{aligned}
& \Theta\left(\int_{0}^{p_{1}}\left[\mathcal{M}\left(p_{1}, \sigma_{1}, \Omega\left(\sigma_{1}\right)\right)-\mathcal{M}\left(p_{1}, \sigma_{1}, Y\left(\sigma_{1}\right)\right)\right] d \sigma_{1},\right. \\
& \left.\cdots, \int_{0}^{p_{k}}\left[\mathcal{M}\left(p_{k}, \sigma_{k}, \Omega\left(\sigma_{k}\right)\right)-\mathcal{M}\left(p_{k}, \sigma_{k}, Y\left(\sigma_{k}\right)\right)\right] d \sigma_{k}, \vartheta \vec{\mu}\right) \\
& =\Theta\left(\lim _{\|\Delta \bar{y}\| \rightarrow 0} \sum_{j=1}^{k}\left[\mathcal{M}\left(p_{1}, \bar{y}_{j}, \Omega\left(\bar{y}_{j}\right)\right)-\mathcal{M}\left(p_{1}, \bar{y}_{j}, Y\left(\bar{y}_{j}\right)\right)\right] \Delta \bar{y}_{i},\right. \\
& \left.\cdots, \lim _{\|\Delta \bar{y}\| \rightarrow 0} \sum_{j=1}^{k}\left[\mathcal{M}\left(p_{k}, \bar{y}_{j}, \Omega\left(\bar{y}_{j}\right)\right)-\mathcal{M}\left(p_{k}, \bar{y}_{j}, Y\left(\bar{y}_{j}\right)\right)\right] \Delta \bar{y}_{i}, \vartheta \vec{\mu}\right)
\end{aligned}
$$




$$
\begin{aligned}
& =\lim _{\|\Delta \bar{y}\| \rightarrow 0} \Theta\left(\sum_{j=1}^{k}\left[\mathcal{M}\left(p_{1}, \bar{y}_{j}, \omega\left(\bar{y}_{j}\right)\right)-\mathcal{M}\left(p_{1}, \bar{y}_{j}, \mathrm{Y}\left(\bar{y}_{j}\right)\right)\right] \Delta \bar{y}_{i},\right. \\
& \left.\cdots, \sum_{j=1}^{k}\left[\mathcal{M}\left(p_{k}, \bar{y}_{j}, \omega\left(\bar{y}_{j}\right)\right)-\mathcal{M}\left(p_{k}, \bar{y}_{j}, \mathrm{Y}\left(\bar{y}_{j}\right)\right)\right] \Delta \bar{y}_{i}, \vartheta \vec{\mu}\right) \\
& \succeq \lim _{\|\Delta \bar{y}\| \rightarrow 0} \circledast_{M} \Theta\left(\left[\mathcal{M}\left(p_{1}, \bar{y}_{j}, \Omega\left(\bar{y}_{j}\right)\right)-\mathcal{M}\left(p_{1}, \bar{y}_{j}, \mathrm{Y}\left(\bar{y}_{j}\right)\right)\right] \Delta \bar{y}_{i},\right. \\
& \left.\cdots,\left[\mathcal{M}\left(p_{k}, \bar{y}_{j}, \Omega\left(\bar{y}_{j}\right)\right)-\mathcal{M}\left(p_{k}, \bar{y}_{j}, \mathrm{Y}\left(\bar{y}_{j}\right)\right)\right] \Delta \bar{y}_{i}, \frac{\vartheta \vec{\mu}}{k}\right) \\
& \succeq \inf _{\xi_{1}, \cdots, \bar{\xi}_{k} \in E_{1}} \Theta\left(\mathcal{M}\left(p_{1}, \xi_{1}, \Omega\left(\xi_{1}\right)\right)-\mathcal{M}\left(p_{1}, \xi_{1}, Y\left(\xi_{1}\right)\right),\right. \\
& \left.\cdots, \mathcal{M}\left(p_{k}, \xi_{k}, \Omega\left(\xi_{k}\right)\right)-\mathcal{M}\left(p_{k}, \xi_{k}, Y\left(\xi_{k}\right)\right), \frac{k \vartheta \vec{\mu}}{k P}\right) \\
& \succeq \inf _{\xi_{1}, \cdots, \bar{\xi}_{k} \in E_{1}} \Theta\left(\Omega\left(\xi_{1}\right)-\mathrm{Y}\left(\xi_{1}\right), \cdots, \Omega\left(\xi_{k}\right)-\mathrm{Y}\left(\xi_{k}\right), \frac{k L_{2} \vartheta \vec{\mu}}{k P}\right) \\
& \succeq \inf _{\xi_{1}, \cdots, \bar{\xi}_{k} \in E_{1}} \phi\left(\xi_{1}, \cdots, \xi_{k}, \frac{P \vec{\mu}}{L_{2}}\right) \\
& \succeq \phi\left(p_{1}, \cdots, p_{k}, L_{2} L_{3} \vec{\mu}\right) .
\end{aligned}
$$

Step 3. Using (7) and (13), we get:

$$
\begin{aligned}
& \Theta\left(\mathcal{I}_{0}^{\kappa, \psi}\left(S\left(\sigma_{1}, \Omega\left(\sigma_{1}\right)\right)-S\left(\sigma_{1}, Y\left(\sigma_{1}\right)\right)\right),\right. \\
& \left.\cdots, 0 \mathcal{I}_{p_{k}}^{\kappa, \psi}\left(S\left(\sigma_{k}, \Omega\left(\sigma_{k}\right)\right)-S\left(\sigma_{k}, Y\left(\sigma_{k}\right)\right)\right), \vartheta \vec{\mu}\right) \quad \succeq \phi\left(p_{1}, \cdots, p_{k}, L_{1} L_{4} \vec{\mu}\right) .
\end{aligned}
$$

Step 4. Using (7) and (14), we get:

$$
\begin{aligned}
& \Theta\left(\mathcal{I}_{0}^{\kappa, \psi}\left(\int_{0}^{\sigma_{1}}\left[\mathcal{M}\left(\sigma_{1}, \varepsilon_{1}, \Omega\left(\varepsilon_{1}\right)\right)-\mathcal{M}\left(\sigma_{1}, \varepsilon_{1}, \mathrm{Y}\left(\varepsilon_{1}\right)\right)\right] d \varepsilon_{1}\right),\right. \\
&\left.\cdots,{ }_{, 0} \mathcal{I}_{p_{k}}^{\kappa, \psi}\left(\int_{0}^{\sigma_{k}}\left[\mathcal{M}\left(\sigma_{k}, \varepsilon_{k}, \Omega\left(\varepsilon_{k}\right)\right)-\mathcal{M}\left(\sigma_{k}, \varepsilon_{k}, Y\left(\varepsilon_{k}\right)\right)\right] d \varepsilon_{k}\right), \vartheta \vec{\mu}\right) \succeq \phi\left(p, L_{2} L_{3} L_{4} \vec{\mu}\right) .
\end{aligned}
$$

From (12)-(16), we have:

$$
\begin{gathered}
\Theta\left({ }_{0} \mathcal{D}_{p_{1}}^{\kappa, \tau, \psi} \Lambda\left(\Omega\left(p_{1}\right)\right)-{ }_{0} \mathcal{D}_{p_{1}}^{\kappa, \tau, \psi} \Lambda\left(\mathrm{Y}\left(p_{1}\right)\right), \cdots,{ }_{0} \mathcal{D}_{p_{k}}^{\kappa, \tau, \psi} \Lambda\left(\Omega\left(p_{k}\right)\right)-{ }_{0} \mathcal{D}_{p_{k}}^{\kappa, \tau, \psi} \Lambda\left(\mathrm{Y}\left(p_{k}\right)\right), 2 \vartheta \vec{\mu}\right) \circledast \\
\Theta\left(\Lambda\left(\Omega\left(p_{1}\right)\right)-\Lambda\left(\mathrm{Y}\left(p_{1}\right)\right), \cdots, \Lambda\left(\Omega\left(p_{k}\right)\right)-\Lambda\left(\mathrm{Y}\left(p_{k}\right)\right), 2 \vartheta \vec{\mu}\right) \\
\succeq \phi\left(p_{1}, \cdots, p_{k}, L_{1} \vec{\mu}\right) \circledast \phi\left(p_{1}, \cdots, p_{k}, L_{2} L_{3} \vec{\mu}\right) \circledast \phi\left(p_{1}, \cdots, p_{k}, L_{1} L_{4} \vec{\mu}\right) \circledast \phi\left(p_{1}, \cdots, p_{k}, L_{2} L_{3} L_{4} \vec{\mu}\right) \\
\succeq \phi\left(p_{1}, \cdots, p_{k}, N_{1} \vec{\mu}\right) \\
\text { where in } N_{1}=\min \left[L_{1}, L_{2} L_{3}, L_{1} L_{4}, L_{2} L_{3} L_{4}\right] . \\
\text { Therefore, } \\
\Theta\left({ }_{0} \mathcal{D}_{p_{1}, \tau, \psi}^{\kappa, \tau}\left(\Omega\left(p_{1}\right)\right)-{ }_{0} \mathcal{D}_{p_{1}}^{\kappa, \tau, \psi} \Lambda\left(\mathrm{Y}\left(p_{1}\right)\right), \cdots,{ }_{0} \mathcal{D} p_{k}{ }^{\kappa, \tau, \psi} \Lambda\left(\Omega\left(p_{k}\right)\right)-{ }_{0} \mathcal{D}_{p_{k}}^{\kappa, \tau, \psi} \Lambda\left(\mathrm{Y}\left(p_{k}\right)\right), 2 \vartheta \vec{\mu}\right) \\
\circledast \Theta\left(\Lambda\left(\Omega\left(p_{1}\right)\right)-\Lambda\left(\mathrm{Y}\left(p_{1}\right)\right), \cdots, \Lambda\left(\Omega\left(p_{k}\right)\right)-\Lambda\left(\mathrm{Y}\left(p_{k}\right)\right), 2 \vartheta \vec{\mu}\right) \\
\succeq \phi\left(p_{1}, \cdots, p_{k}, N_{4} \vec{\mu}\right),
\end{gathered}
$$


where $N_{4}=\frac{1}{2} \min \left[L_{1}, L_{2} L_{3}, L_{1} L_{4}, L_{2} L_{3} L_{4}\right]$ and implying that:

$$
d(\Lambda(\Omega), \Lambda(Y)) \leq \frac{\vartheta}{N_{4}} .
$$

Therefore,

$$
d(\Lambda(\Omega), \Lambda(Y)) \leq \frac{1}{N_{4}} d(\Omega, Y) .
$$

Thus, $\Lambda$ with Lipschitz constant $\frac{1}{N_{4}}$ is a strictly contractive mapping. Let $\omega \in B$. We show that $d(\Lambda(\omega), \omega)<\infty$. Using (7) and (8), we get:

$$
\begin{aligned}
& \Theta\left({ }_{0} \mathcal{D}_{p_{1}}^{\kappa, \tau, \psi}\left[\Lambda\left(\omega\left(p_{1}\right)\right)-\omega\left(p_{1}\right)\right], \cdots{ }_{0} \mathcal{D}_{p_{k}}^{\kappa, \tau, \psi}\left[\Lambda\left(\omega\left(p_{k}\right)\right)-\omega\left(p_{k}\right)\right], \vec{\mu}\right) \\
& \circledast \Theta\left(\Lambda\left(\omega\left(p_{1}\right)\right)-\omega\left(p_{1}\right), \cdots, \Lambda\left(\omega\left(p_{k}\right)\right)-\omega\left(p_{k}\right), \vec{\mu}\right) \\
& =\Theta\left(\boldsymbol{S}\left(p_{1}, \omega\left(p_{1}\right)\right)+\int_{0}^{p_{1}} \mathcal{M}\left(p_{1}, \sigma_{1}, \omega\left(\sigma_{1}\right)\right) d \sigma_{1}-{ }_{0} \mathcal{D}_{p_{1}}^{\kappa, \tau, \psi} \omega\left(p_{1}\right),\right. \\
& \left.\cdots, \boldsymbol{S}\left(p_{k}, \omega\left(p_{k}\right)\right)+\int_{0}^{p_{k}} \mathcal{M}\left(p_{k}, \sigma_{k}, \omega\left(\sigma_{k}\right)\right) d \sigma_{k}-{ }_{0} \mathcal{D}_{p_{k}}^{\kappa, \tau, \psi} \omega\left(p_{k}\right), \vec{\mu}\right) \\
& \circledast \Theta\left(\mathcal{I}_{0}^{\kappa, \psi}\left(S\left(p_{1}, \omega\left(p_{1}\right)\right)\right)+{ }_{0} \mathcal{I}_{p_{1}}^{\kappa, \psi}\left(\int_{0}^{p_{1}} \mathcal{M}\left(p_{1}, \sigma_{1}, \omega\left(\sigma_{1}\right)\right) d \sigma_{1}\right)-{ }_{0} \mathcal{I}_{p_{1}}^{\kappa, \psi}{ }_{0} \mathcal{D}_{p_{1}}^{\kappa, \tau, \psi} \omega\left(p_{1}\right),\right. \\
& \left.\cdots,{ }_{0} \mathcal{I}_{p_{k}}^{\kappa, \psi}\left(S\left(p_{k}, \omega\left(p_{k}\right)\right)\right)+{ }_{0} \mathcal{I}_{p_{k}}^{\kappa, \psi}\left(\int_{0}^{p_{k}} \mathcal{M}\left(p_{k}, \sigma_{k}, \omega\left(\sigma_{k}\right)\right) d \sigma_{k}\right)-{ }_{0} \mathcal{I}_{p_{k}}^{\kappa, \psi}{ }_{0} \mathcal{D}_{p_{k}}^{\kappa, \tau, \psi} \omega\left(p_{k}\right), \vec{\mu}\right) \\
& =\Theta\left(\boldsymbol{S}\left(p_{1}, \omega\left(p_{1}\right)\right)+\int_{0}^{p_{1}} \mathcal{M}\left(p_{1}, \sigma_{1}, \omega\left(\sigma_{1}\right)\right) d \sigma_{1}-{ }_{0} \mathcal{D}_{p_{1}}^{\kappa, \tau, \psi} \omega\left(p_{1}\right),\right. \\
& \left.\cdots, \boldsymbol{S}\left(p_{k}, \omega\left(p_{k}\right)\right)+\int_{0}^{p_{k}} \mathcal{M}\left(p_{k}, \sigma_{k}, \omega\left(\sigma_{k}\right)\right) d \sigma_{k}-{ }_{0} \mathcal{D}_{p_{k}}^{\kappa, \tau, \psi} \omega\left(p_{k}\right), \vec{\mu}\right) \\
& \circledast \Theta\left(\mathcal{I}_{0}^{\kappa, \psi}\left[\boldsymbol{S}\left(p_{1}, \omega\left(p_{1}\right)\right)+\left(\int_{0}^{p_{1}} \mathcal{M}\left(p_{1}, \sigma_{1}, \omega\left(\sigma_{1}\right)\right) d \sigma_{1}\right)-{ }_{0} \mathcal{D}_{p_{1}}^{\kappa, \tau, \psi} \omega\left(p_{1}\right)\right],\right. \\
& \left.\cdots{ }_{, 0} \mathcal{I}_{p_{k}}^{\kappa, \psi}\left[\boldsymbol{S}\left(p_{k}, \omega\left(p_{k}\right)\right)+\left(\int_{0}^{p_{k}} \mathcal{M}\left(p_{k}, \sigma_{k}, \omega\left(\sigma_{k}\right)\right) d \sigma_{k}\right)-{ }_{0} \mathcal{D}_{p_{k}}^{\kappa, \tau} \omega\left(p_{k}\right)\right], \vec{\mu}\right) \\
& \succeq \phi\left(p_{1}, \cdots, p_{k}, \vec{\mu}\right) \circledast \phi\left(p_{1}, \cdots, p_{k}, L_{4} \vec{\mu}\right) \\
& \succeq \phi\left(p_{1}, \cdots, p_{k}, N_{2} \vec{\mu}\right) \text {, }
\end{aligned}
$$

for every $\vec{\mu} \in\left(E_{2}\right)^{n}$ and $N_{2}=\min \left[1, L_{4}\right]$. Then, we have $d(\Lambda(\omega), \omega)<\frac{1}{N_{2}}<\infty$.

Now, Theorem 1 enables us to find an element $\omega_{0}$ in $B$ satisfying the following: (1) $\omega_{0}$ is a fixed point of $\Lambda$, i.e.,

$$
\begin{aligned}
\omega_{0}(p) & =\Lambda\left(\omega_{0}(p)\right) \\
& ={ }_{0} \mathcal{I}_{p}^{\kappa, \psi}\left(\boldsymbol{S}\left(\sigma, \omega_{0}(\sigma)\right)\right)+{ }_{0} \mathcal{I}_{p}^{\kappa, \psi}\left(\int_{0}^{\sigma} \mathcal{M}\left(\sigma, \varepsilon, \omega_{0}(\varepsilon)\right) d \varepsilon\right),
\end{aligned}
$$

which is unique in the set:

$$
B^{*}=\{\Omega \in B: d(\Lambda(\omega), \Omega)<\infty\} .
$$

Take $_{0} \mathcal{D}_{p}^{\kappa, \tau, \psi}$ from (22). We get:

$$
{ }_{0} \mathcal{D}_{p}^{\kappa, \tau, \psi} \omega_{0}(p)=S\left(p, \omega_{0}(p)\right)+\int_{0}^{p} \mathcal{M}\left(p, \sigma, \omega_{0}(\sigma)\right) d \sigma,
$$

where $\kappa \in E_{5}{ }^{\circ}, S: E_{1} \times V \rightarrow V, \mathcal{M}: E_{1} \times E_{1} \times V \rightarrow V$. 
(2) $d\left(\Lambda^{k}(\omega), \omega_{0}\right) \rightarrow 0$ as $n \rightarrow \infty$;

(3) $d\left(\omega, \omega_{0}\right) \leq N_{3} d(\Lambda(\omega), w) \leq \frac{N_{3}}{N_{2}}$,

where $N_{2}=\min \left[1, L_{4}\right]$ and $N_{3}=\frac{\min \left[L_{1}, L_{2} L_{3}, L_{1} L_{4}, L_{2} L_{3} L_{4}\right]}{\min \left[L_{1}, L_{2} L_{3}, L_{1} L_{4}, L_{2} L_{3} L_{4}\right]-2}$ and implying that:

$$
\begin{gathered}
\Theta\left({ }_{0} \mathcal{D}_{p_{1}}^{\kappa, \tau, \psi} \omega\left(p_{1}\right)-{ }_{0} \mathcal{D}_{p_{1}}^{\kappa, \tau, \psi} \omega_{0}\left(p_{1}\right), \cdots,{ }_{0} \mathcal{D}_{p_{k}}^{\kappa, \tau, \psi} \omega\left(p_{k}\right)-{ }_{0} \mathcal{D}_{p_{k}}^{\kappa, \tau, \psi} \omega_{0}\left(p_{k}\right), \vec{\mu}\right) \circledast \\
\Theta\left(\omega\left(p_{1}\right)-\omega_{0}\left(p_{1}\right), \cdots, \omega\left(p_{k}\right)-\omega_{0}\left(p_{k}\right), \vec{\mu}\right) \\
\succeq \phi\left(p_{1}, \cdots, p_{k}, \frac{N_{2}}{N_{3}} \vec{\mu}\right),
\end{gathered}
$$

for every $p_{1}, \cdots, p_{k} \in E_{1}$ and $\vec{\mu} \in\left(E_{2}\right)^{n}$.

Now, we prove that the fixed point in $B^{*}$ is unique. Suppose that $\varsigma_{0}$ is an element of $B$ satisfying (9) and (10). We prove that $\varsigma_{0}=\omega_{0}$ and $\varsigma_{0} \in B^{*}$. From (9), we get:

$$
{ }_{0} \mathcal{D}_{p}^{\kappa, \tau, \psi} \varsigma_{0}(p)=S\left(p, \varsigma_{0}(p)\right)+\int_{0}^{p} \mathcal{M}\left(p, \sigma, \varsigma_{0}(\sigma)\right) d \sigma,
$$

and so:

$$
\begin{aligned}
\varsigma_{0}(p) & ={ }_{0} \mathcal{I}_{p}^{\kappa, \psi} \boldsymbol{S}\left(\sigma, \varsigma_{0}(\sigma)\right)+{ }_{0} \mathcal{I}_{p}^{\kappa, \psi} \int_{0}^{\sigma} \mathcal{M}\left(\sigma, \varepsilon, \varsigma_{0}(\varepsilon)\right) d \varepsilon \\
& =\Lambda\left(\varsigma_{0}(p)\right),
\end{aligned}
$$

where $\kappa \in E_{5}^{\circ}, S: E_{1} \times V \rightarrow V, \mathcal{M}: E_{1} \times E_{1} \times V \rightarrow V$.

Now, we show that:

$$
\varsigma_{0} \in\{\Omega \in B: d(\Lambda(\omega), \Omega)<\infty\},
$$

i.e., $d\left(\Lambda(\omega), \varsigma_{0}\right)<\infty$. We set $j=\frac{\min \left[1, L_{4}\right]\left(\min \left[L_{1}, L_{2} L_{3}, L_{1} L_{4}, L_{2} L_{3} L_{4}\right]-2\right)}{\min \left[L_{1}, L_{2} L_{3}, L_{1} L_{4}, L_{2} L_{3} L_{4}\right]}$. From (10), we get:

$$
\begin{gathered}
\Theta\left({ }_{0} \mathcal{D}_{p_{1}}^{\kappa, \tau, \psi} \omega\left(p_{1}\right)-{ }_{0} \mathcal{D}_{p_{1}}^{\kappa, \tau, \psi} \varsigma_{0}\left(p_{1}\right), \cdots,{ }_{0} \mathcal{D}_{p_{k}}^{\kappa, \tau, \psi} \omega\left(p_{k}\right)-{ }_{0} \mathcal{D}_{p_{k}}^{\kappa, \tau} \varsigma_{0}\left(p_{k}\right), \vec{\mu}\right) \circledast \\
\Theta\left(\omega\left(p_{1}\right)-\varsigma_{0}\left(p_{1}\right), \cdots, \omega\left(p_{k}\right)-\varsigma_{0}\left(p_{k}\right), \vec{\mu}\right) \succeq \phi\left(p_{1}, \cdots, p_{k}, \jmath \vec{\mu}\right),
\end{gathered}
$$

for every $p_{1}, \cdots, p_{k} \in E_{1}$ and $\vec{\mu} \in\left(E_{2}\right)^{n}$.

From (4) and (27), we get:

$$
\begin{gathered}
\Theta\left(S\left(p_{1}, \omega\left(p_{1}\right)\right)-\boldsymbol{S}\left(p_{1}, \varsigma_{0}\left(p_{1}\right)\right), \cdots, \boldsymbol{S}\left(p_{k}, \omega\left(p_{k}\right)\right)-\boldsymbol{S}\left(p_{k}, \varsigma_{0}\left(p_{k}\right)\right), \vec{\mu}\right) \\
\succeq \Theta\left(\omega\left(p_{1}\right)-\varsigma_{0}\left(p_{1}\right), \cdots, \omega\left(p_{k}\right)-\varsigma_{0}\left(p_{k}\right), L_{1} \vec{\mu}\right) \\
\left.\succeq \phi\left(p_{1}, \cdots, p_{k}, L_{1}\right] \vec{\mu}\right) .
\end{gathered}
$$

Furthermore, from (5) and (27), we get:

$$
\begin{gathered}
\Theta\left(\mathcal{M}\left(p_{1}, \sigma_{1}, \omega\left(\sigma_{1}\right)\right)-\mathcal{M}\left(p_{1}, \sigma_{1}, \varsigma_{0}\left(\sigma_{1}\right)\right), \cdots, \mathcal{M}\left(p_{k}, \sigma_{k}, \omega\left(\sigma_{k}\right)\right)-\mathcal{M}\left(p_{k}, \sigma_{k}, \varsigma_{0}\left(\sigma_{k}\right)\right), \vec{\mu}\right) \\
\succeq \Theta\left(\omega\left(\sigma_{1}\right)-\varsigma_{0}\left(\sigma_{1}\right), \cdots, \omega\left(\sigma_{k}\right)-\varsigma_{0}\left(\sigma_{k}\right), L_{2} \vec{\mu}\right) \\
\left.\succeq \phi\left(p_{1}, \cdots, p_{k}, L_{2}\right] \vec{\mu}\right), \\
\text { for every } p_{1}, \cdots, p_{k} \in E_{1}, \sigma_{i} \preceq p_{i}(i=1,2, \cdots, k), \text { and } \vec{\mu} \in\left(E_{2}\right)^{n} .
\end{gathered}
$$


Now, using Step 2 and (29), we get:

$$
\begin{gathered}
\Theta\left(\int_{0}^{p_{1}}\left[\mathcal{M}\left(p_{1}, \sigma_{1}, \omega\left(\sigma_{1}\right)\right)-\mathcal{M}\left(p_{1}, \sigma_{1}, \varsigma_{0}\left(\sigma_{1}\right)\right)\right] d \sigma_{1}, \cdots,\right. \\
\left.\int_{0}^{p_{k}}\left[\mathcal{M}\left(p_{k}, \sigma_{k}, \omega\left(\sigma_{k}\right)\right)-\mathcal{M}\left(p_{k}, \sigma_{k}, \varsigma_{0}\left(\sigma_{k}\right)\right)\right] d \sigma_{k}, \vec{\mu}\right) \\
\succeq \phi\left(p_{1}, \cdots, p_{k}, L_{2} L_{3} \vec{\mu}\right) \\
\succeq \phi\left(p_{1}, \cdots, p_{k}, L_{2} L_{3} j \vec{\mu}\right) .
\end{gathered}
$$

Using the triangular inequality (MVF-K-N3), (28), and (30), we get:

$$
\begin{gathered}
\Theta\left(\boldsymbol{S}\left(p_{1}, \omega\left(p_{1}\right)\right)-\boldsymbol{S}\left(p_{1}, \varsigma_{0}\left(p_{1}\right)\right)+\int_{0}^{p_{1}}\left[\mathcal{M}\left(p_{1}, \sigma_{1}, \omega\left(\sigma_{1}\right)\right)-\mathcal{M}\left(p_{1}, \sigma_{1}, \varsigma_{0}\left(\sigma_{1}\right)\right)\right] d \sigma_{1}, \cdots,\right. \\
\left.\boldsymbol{S}\left(p_{k}, \omega\left(p_{k}\right)\right)-\boldsymbol{S}\left(p_{k}, \varsigma_{0}\left(p_{k}\right)\right)+\int_{0}^{p_{k}}\left[\mathcal{M}\left(p_{k}, \sigma_{k}, \omega\left(\sigma_{k}\right)\right)-\mathcal{M}\left(p_{k}, \sigma_{k}, \varsigma_{0}\left(\sigma_{k}\right)\right)\right] d \sigma_{k}, 2 \vec{\mu}\right) \\
\succeq \Theta\left(\boldsymbol{S}\left(p_{1}, \omega\left(p_{1}\right)\right)-\boldsymbol{S}\left(p_{1}, \varsigma_{0}\left(p_{1}\right)\right), \cdots, \boldsymbol{S}\left(p_{k}, \omega\left(p_{k}\right)\right)-\boldsymbol{S}\left(p_{k}, \varsigma_{0}\left(p_{k}\right)\right), \vec{\mu}\right) \\
\circledast \Theta\left(\int_{0}^{p_{1}}\left[\mathcal{M}\left(p_{1}, \sigma_{1}, \omega\left(\sigma_{1}\right)\right)-\mathcal{M}\left(p_{1}, \sigma_{1}, \varsigma_{0}\left(\sigma_{1}\right)\right)\right] d \sigma_{1}, \cdots,\right. \\
\left.\int_{0}^{p_{k}}\left[\mathcal{M}\left(p_{k}, \sigma_{k}, \omega\left(\sigma_{k}\right)\right)-\mathcal{M}\left(p_{k}, \sigma_{k}, \varsigma_{0}\left(\sigma_{k}\right)\right)\right] d \sigma_{k}, \vec{\mu}\right) \\
\left.\left.\succeq \phi\left(p_{1}, \cdots, p_{k}, L_{1}\right] \vec{\mu}\right) \circledast \phi\left(p_{1}, \cdots, p_{k}, L_{2} L_{3}\right] \vec{\mu}\right) \\
\left.\succeq \phi\left(p_{1}, \cdots, p_{k}, N_{5}\right] \vec{\mu}\right),
\end{gathered}
$$

where in $N_{5}=\min \left[L_{1}, L_{2} L_{3}\right]$, and so:

$$
\begin{gathered}
\Theta\left(\boldsymbol{S}\left(p_{1}, \omega\left(p_{1}\right)\right)-\boldsymbol{S}\left(p_{1}, \varsigma_{0}\left(p_{1}\right)\right)+\int_{0}^{p_{1}}\left[\mathcal{M}\left(p_{1}, \sigma_{1}, \omega\left(\sigma_{1}\right)\right)-\mathcal{M}\left(p_{1}, \sigma_{1}, \varsigma_{0}\left(\sigma_{1}\right)\right)\right] d \sigma_{1}, \cdots,\right. \\
\left.\boldsymbol{S}\left(p_{k}, \omega\left(p_{k}\right)\right)-\boldsymbol{S}\left(p_{k}, \varsigma_{0}\left(p_{k}\right)\right)+\int_{0}^{p_{k}}\left[\mathcal{M}\left(p_{k}, \sigma_{k}, \omega\left(\sigma_{k}\right)\right)-\mathcal{M}\left(p_{k}, \sigma_{k}, \varsigma_{0}\left(\sigma_{k}\right)\right)\right] d \sigma_{k}, \vec{\mu}\right) \\
\left.\succeq \phi\left(p_{1}, \cdots, p_{k}, N_{6}\right] \vec{\mu}\right),
\end{gathered}
$$

where $N_{6}=\frac{1}{2} \min \left[L_{1}, L_{2} L_{3}\right]$

We apply (7) and get:

$$
\begin{gathered}
\Theta\left({ }_{0} \mathcal{I}_{p_{1}}^{\kappa, \psi}\left[S\left(\sigma_{1}, \omega\left(\sigma_{1}\right)\right)-\boldsymbol{S}\left(\sigma_{1}, \varsigma_{0}\left(\sigma_{1}\right)\right)\right]+{ }_{0} \mathcal{I}_{p_{1}}^{\kappa, \psi} \int_{0}^{\sigma_{1}}\left[\mathcal{M}\left(\sigma_{1}, \varepsilon_{1}, \omega\left(\varepsilon_{1}\right)\right)-\mathcal{M}\left(\sigma_{1}, \varepsilon_{1}, \varsigma_{0}\left(\varepsilon_{1}\right)\right)\right] d \varepsilon_{1},\right. \\
\left.\ldots, \mathcal{I}_{p_{k}}^{\kappa, \psi}\left[S(\sigma, \omega(\sigma))-\boldsymbol{S}\left(\sigma, \varsigma_{0}(\sigma)\right)\right]+{ }_{0} \mathcal{I}_{p_{k}}^{\kappa, \psi} \int_{0}^{\sigma}\left[\mathcal{M}(\sigma, \varepsilon, \omega(\varepsilon))-\mathcal{M}\left(\sigma, \varepsilon, \varsigma_{0}(\varepsilon)\right)\right] d \varepsilon, \vec{\mu}\right) \\
\left.\succeq \phi\left(p_{1}, \cdots, p_{k}, L_{4} N_{6}\right] \vec{\mu}\right),
\end{gathered}
$$

for every $p_{1}, \cdots, p_{k} \in E_{1}$ and $\vec{\mu} \in\left(E_{2}\right)^{n}$, and $N_{6}=\frac{1}{2} \min \left[L_{1}, L_{2} L_{3}\right]$.

Using (32) and (33), we get: 


$$
\begin{gathered}
\Theta\left({ }_{0} \mathcal{D}_{p_{1}}^{\kappa, \tau, \psi}\left[\Lambda\left(\omega\left(p_{1}\right)\right)-\varsigma_{0}\left(p_{1}\right)\right], \cdots,{ }_{0} \mathcal{D}_{p_{k}}^{\kappa, \tau, \psi}\left[\Lambda\left(\omega\left(p_{k}\right)\right)-\varsigma_{0}\left(p_{k}\right)\right], \vec{\mu}\right) \\
\circledast \Theta\left(\Lambda\left(\omega\left(p_{1}\right)\right)-\varsigma_{0}\left(p_{1}\right), \cdots, \Lambda\left(\omega\left(p_{k}\right)\right)-\varsigma_{0}\left(p_{k}\right), \vec{\mu}\right) \\
=\Theta\left(S\left(p_{1}, \omega\left(p_{1}\right)\right)-S\left(p_{1}, \varsigma_{0}\left(p_{1}\right)\right)+\int_{0}^{p_{1}}\left[\mathcal{M}\left(p_{1}, \sigma_{1}, \omega\left(\sigma_{1}\right)\right)-\mathcal{M}\left(p_{1}, \sigma_{1}, \varsigma_{0}\left(\sigma_{1}\right)\right)\right] d \sigma_{1}, \cdots,\right. \\
\left.\boldsymbol{S}\left(p_{k}, \omega\left(p_{k}\right)\right)-\boldsymbol{S}\left(p_{k}, \varsigma_{0}\left(p_{k}\right)\right)+\int_{0}^{p_{k}}\left[\mathcal{M}\left(p_{k}, \sigma_{k}, \omega\left(\sigma_{k}\right)\right)-\mathcal{M}\left(p_{k}, \sigma_{k}, \varsigma_{0}\left(\sigma_{k}\right)\right)\right] d \sigma_{k}, \vec{\mu}\right) \\
\circledast \Theta\left({ }_{0} \mathcal{I}_{p_{1}}^{\kappa, \psi}\left[S\left(\sigma_{1}, \omega\left(\sigma_{1}\right)\right)-S\left(\sigma_{1}, \varsigma_{0}\left(\sigma_{1}\right)\right)\right]+{ }_{0} \mathcal{I}_{p_{1}}^{\kappa, \psi} \int_{0}^{\sigma_{1}}\left[\mathcal{M}\left(\sigma_{1}, \varepsilon_{1}, \omega\left(\varepsilon_{1}\right)\right)-\mathcal{M}\left(\sigma_{1}, \varepsilon_{1}, \varsigma_{0}\left(\varepsilon_{1}\right)\right)\right] d \varepsilon_{1}, \cdots,\right. \\
\left.{ }_{0} \mathcal{I}_{p_{k}}^{\kappa, \psi}\left[S\left(\sigma_{k}, \omega\left(\sigma_{k}\right)\right)-\boldsymbol{S}\left(\sigma_{k}, \varsigma_{0}\left(\sigma_{k}\right)\right)\right]+{ }_{0} \mathcal{I}_{p_{k}}^{\kappa, \psi} \int_{0}^{\sigma_{k}}\left[\mathcal{M}\left(\sigma_{k}, \varepsilon_{k}, \omega\left(\varepsilon_{k}\right)\right)-\mathcal{M}\left(\sigma_{k}, \varepsilon_{k}, \varsigma_{0}\left(\varepsilon_{k}\right)\right)\right] d \varepsilon_{k}, \vec{\mu}\right) \\
\left.\left.\succeq \phi\left(p_{1}, \cdots, p_{k}, N_{6}\right] \vec{\mu}\right) \circledast \phi\left(p_{1}, \cdots, p_{k}, L_{4} N_{6}\right] \vec{\mu}\right) \\
\succeq \phi\left(p_{1}, \cdots, p_{k}, \frac{\left.\left(L_{4}+1\right) N_{6}\right] \vec{\mu}}{L_{4}}\right),
\end{gathered}
$$

which $N_{6}=\frac{1}{2} \min \left[L_{1}, L_{2} L_{3}\right]$, which implies that:

$$
d\left(\Lambda(\omega), \varsigma_{0}\right) \leq \frac{L_{4}}{\jmath\left(L_{4}+1\right) N_{6}}<\infty
$$

then $\varsigma_{0} \in B^{*}$.

\section{Best Approximation of $\psi$-Hilfer Fractional Volterra Integral Equation}

Now, we are ready to study the Hyers-Ulam-Rassias stability of the $\psi$-Hilfer fractional Volterra integral equation:

$$
\Omega(p)=S(p, \Omega(p))+{ }_{0} \mathcal{I}_{p}^{\kappa, \psi} \mathcal{M}(p, \sigma, \Omega(\sigma)),
$$

where $\kappa \in E_{5}{ }^{\circ}, S: E_{1} \times V \rightarrow V, \mathcal{M}: E_{1} \times E_{1} \times V \rightarrow V$ and get the best approximation with a better estimate for the pseudo $\psi$-Hilfer fractional Volterra integral equation. Our method can be used for new problems; for more problems and details, we refer to [17-34].

Theorem 4. Let $(V, \Theta, \circledast)$ be an MVFB-space and $L_{1}, L_{2}, L_{3}, L_{4}$ and $T$ be positive constant such that $N_{1}>2$ where $N_{1}=\min \left[L_{1}, L_{2} L_{3}, L_{1} L_{4}, L_{2} L_{3} L_{4}\right]$. Assume that the continuous mappings $S: E_{1} \times V \rightarrow V, \mathcal{M}: E_{1} \times E_{1} \times V \rightarrow V$ with MVFF $\phi:\left(E_{1}\right)^{k} \times\left(E_{2}\right)^{n} \rightarrow \operatorname{diag}\left(M_{n}\left(E_{3}\right)\right)$ satisfying (4)-(7).

Let $\omega: E_{1} \rightarrow V$ be a differentiable function satisfying:

$$
\begin{gathered}
\Theta\left(\omega\left(p_{1}\right)-S\left(p_{1}, \omega\left(p_{1}\right)\right)-{ }_{0} \mathcal{I}_{p_{1}}^{\kappa, \psi} \mathcal{M}\left(p_{1}, \sigma_{1}, \omega\left(\sigma_{1}\right)\right), \cdots,\right. \\
\left.\omega\left(p_{k}\right)-S\left(p_{k}, \omega\left(p_{k}\right)\right)-{ }_{0} \mathcal{I}_{p_{k}}^{\kappa, \psi} \mathcal{M}\left(p_{k}, \sigma_{k}, \omega\left(\sigma_{k}\right)\right), \vec{\mu}\right) \\
\succeq \phi\left(p_{1}, \cdots, p_{k}, \vec{\mu}\right),
\end{gathered}
$$

for every $p_{1}, \cdots, p_{k} \in E_{1}$ and $\vec{\mu} \in\left(E_{2}\right)^{n}$. Then, we are to be able find a unique differentiable function $\omega_{0}: E_{1} \rightarrow V$ such that:

$$
\omega_{0}(p)=S\left(p, \omega_{0}(p)\right)+{ }_{0} \mathcal{I}_{p}^{\kappa, \psi} \mathcal{M}\left(p, \sigma, \omega_{0}(\sigma)\right),
$$


and:

$$
\begin{array}{r}
\Theta\left(\omega\left(p_{1}\right)-\omega_{0}\left(p_{1}\right), \cdots, \omega\left(p_{k}\right)-\omega_{0}\left(p_{k}\right), \vec{\mu}\right) \\
\phi\left(p_{1}, \cdots, p_{k}, \frac{N_{2}}{N_{3}} \vec{\mu}\right),
\end{array}
$$

for every $p_{1}, \cdots, p_{k} \in E_{1}$ and $\vec{\mu} \in\left(E_{2}\right)^{n}$, and $N_{2}=\min \left[1, L_{4}\right]$ and $N_{3}=\frac{\min \left[L_{1}, L_{2} L_{3}, L_{1} L_{4}, L_{2} L_{3} L_{4}\right]}{\min \left[L_{1}, L_{2} L_{3}, L_{1} L_{4}, L_{2} L_{3} L_{4}\right]-2}$.

Proof. We set

$$
B:=\left\{\Omega: E_{1} \rightarrow V, \Omega \text { is differentiable }\right\}
$$

and introduce the $E_{4}$-valued metric on $B$ as,

$$
\begin{array}{r}
d(\Omega, Y)=\inf \left\{C \in E_{6}: \Theta\left(\Omega\left(p_{1}\right)-Y\left(p_{1}\right), \cdots, \Omega\left(p_{k}\right)-Y\left(p_{k}\right), \vec{\mu}\right) \succeq \phi\left(p_{1}, \cdots, p_{k}, \frac{\vec{\mu}}{C}\right),\right. \\
\left.\forall \Omega, Y \in B, p_{1}, \cdots, p_{k} \in E_{1}, \vec{\mu} \in\left(E_{2}\right)^{n}\right\} .
\end{array}
$$

By Theorem 2, we have that $(B, d)$ is a complete $E_{4}$-valued metric space.

Now, we define the mapping $\Lambda$ from $B$ to $B$ by:

$$
\Lambda\left(\Omega\left(p_{i}\right)\right)=S(\sigma, \Omega(\sigma))+{ }_{0} \mathcal{I}_{p_{i}}^{\kappa, \psi}\left(\int_{0}^{\sigma} \mathcal{M}(\sigma, \varepsilon, \Omega(\varepsilon)) d \varepsilon\right),
$$

where $\kappa \in\left(E_{5}\right)^{\circ}, S: E_{1} \times V \rightarrow V, \mathcal{M}: E_{1} \times E_{1} \times V \rightarrow V$, and $p_{i} \in E_{1}(i=1,2, \cdots, k)$. We prove that $\Lambda$ is a strictly contractive mapping. Let $\Omega, Y \in B, C \in E_{6}$ and $d(\Omega, Y)<\vartheta$, then we have:

$$
\begin{array}{r}
\Theta\left(\Omega\left(p_{1}\right)-\mathrm{Y}\left(p_{1}\right), \cdots, \Omega\left(p_{k}\right)-\mathrm{Y}\left(p_{k}\right), \vartheta \vec{\mu}\right) \succeq \phi\left(p_{1}, \cdots, p_{k}, \vec{\mu}\right), \\
\forall \Omega, Y \in B, p_{1}, \cdots, p_{k} \in E_{1}, \vec{\mu} \in\left(E_{2}\right)^{n} .
\end{array}
$$

Using the properties (MVF-K-N2) and (MVF-K-N3) of Definition 2, (4)-(7), and (39), we have:

$$
\begin{gathered}
\Theta\left(\Lambda\left(\Omega\left(p_{1}\right)\right)-\Lambda\left(\mathrm{Y}\left(p_{1}\right)\right), \cdots, \Lambda\left(\Omega\left(p_{k}\right)\right)-\Lambda\left(\mathrm{Y}\left(p_{k}\right)\right), 2 \vartheta \vec{\mu}\right) \\
=\Theta\left(\left[\boldsymbol{S}\left(p_{1}, \Omega\left(p_{1}\right)\right)-\boldsymbol{S}\left(p_{1}, \mathrm{Y}\left(p_{1}\right)\right)\right]+{ }_{0} \mathcal{I}_{p_{1}}^{\kappa, \psi}\left[\mathcal{M}\left(p_{1}, \sigma_{1}, \Omega\left(\sigma_{1}\right)\right)-\mathcal{M}\left(p_{1}, \sigma_{1}, \mathrm{Y}\left(\sigma_{1}\right)\right)\right] d \sigma_{1}, \cdots,\right. \\
\left.\left[S\left(p_{k}, \Omega\left(p_{k}\right)\right)-\boldsymbol{S}\left(p_{k}, \mathrm{Y}\left(p_{k}\right)\right)\right]+{ }_{0} \mathcal{I}_{p_{k}, \psi}^{\kappa, \psi}\left[\mathcal{M}\left(p_{k}, \sigma_{k}, \Omega\left(\sigma_{k}\right)\right)-\mathcal{M}\left(p_{k}, \sigma_{k}, \mathrm{Y}\left(\sigma_{k}\right)\right)\right] d \sigma_{k}, 2 \vartheta \vec{\mu}\right) \\
\succeq \Theta\left(\boldsymbol{S}\left(p_{1}, \Omega\left(p_{1}\right)\right)-\boldsymbol{S}\left(p_{1}, \mathrm{Y}\left(p_{1}\right)\right), \cdots, \boldsymbol{S}\left(p_{k}, \Omega\left(p_{k}\right)\right)-\boldsymbol{S}\left(p_{k}, \mathrm{Y}\left(p_{k}\right)\right), \vartheta \vec{\mu}\right) \\
\circledast \Theta\left(\int_{0}^{p_{1}}\left[\mathcal{M}\left(p_{1}, \sigma_{1}, \Omega\left(\sigma_{1}\right)\right)-\mathcal{M}\left(p_{1}, \sigma_{1}, \mathrm{Y}\left(\sigma_{1}\right)\right)\right] d \sigma_{1}, \cdots,\right. \\
\left.\int_{0}^{p_{k}}\left[\mathcal{M}\left(p_{k}, \sigma_{k}, \Omega\left(\sigma_{k}\right)\right)-\mathcal{M}\left(p_{k}, \sigma_{k}, \mathrm{Y}\left(\sigma_{k}\right)\right)\right] d \sigma_{k}, \vartheta \vec{\mu}\right) \\
\succeq \phi\left(p_{1}, \cdots, p_{k}, L_{1} \vec{\mu}\right) \circledast \phi\left(p_{1}, \cdots, p_{k}, L_{2} L_{4} \vec{\mu}\right) \\
\succeq \phi\left(p_{1}, \cdots, p_{k}, N_{7} \vec{\mu}\right),
\end{gathered}
$$

where in $N_{7}=\min \left[L_{1}, L_{2} L_{4}\right]$, and so:

$$
\begin{gathered}
\Theta\left(\Lambda\left(\Omega\left(p_{1}\right)\right)-\Lambda\left(\mathrm{Y}\left(p_{1}\right)\right), \cdots, \Lambda\left(\Omega\left(p_{k}\right)\right)-\Lambda\left(\mathrm{Y}\left(p_{k}\right)\right), \vartheta \vec{\mu}\right) \\
\succeq \phi\left(p_{1}, \cdots, p_{k}, N_{8} \vec{\mu}\right),
\end{gathered}
$$

for every $p_{1}, \cdots, p_{k} \in E_{1}$ and $\vec{\mu} \in\left(E_{2}\right)^{n}$, and $N_{8}=\frac{1}{2} \min \left[L_{1}, L_{2} L_{4}\right]$. 
Then:

$$
d(\Lambda(\Omega), \Lambda(Y)) \leq \frac{\vartheta}{N_{8}}
$$

and so:

$$
d(\Lambda(\Omega), \Lambda(\mathrm{Y})) \leq \frac{1}{N_{8}} d(\Omega, \mathrm{Y})
$$

Then, $\Lambda$ is a strictly contractive mapping with Lipschitz constant $\frac{1}{N_{8}}$.

Let $\omega \in B$. We show that $d(\Lambda(\omega), \omega)<\infty$. Using (36), we get:

$$
\begin{gathered}
\Theta\left(\Lambda\left(\omega\left(p_{1}\right)\right)-\omega\left(p_{1}\right), \cdots, \Lambda\left(\omega\left(p_{k}\right)\right)-\omega\left(p_{k}\right), \vec{\mu}\right) \\
=\Theta\left(S\left(p_{1}, \omega\left(p_{1}\right)\right)+{ }_{0} \mathcal{I}_{p_{1}}^{\kappa, \psi} \mathcal{M}\left(p_{1}, \sigma_{1}, \omega\left(\sigma_{1}\right)\right) d \sigma_{1}-\omega\left(p_{1}\right),\right. \\
\left.\cdots, S\left(p_{k}, \omega\left(p_{k}\right)\right)+{ }_{0} \mathcal{I}_{p_{k}}^{\kappa, \psi} \mathcal{M}\left(p_{k}, \sigma_{k}, \omega\left(\sigma_{k}\right)\right) d \sigma_{k}-\omega\left(p_{k}\right), \vec{\mu}\right) \\
\succeq \phi\left(p_{1}, \cdots, p_{k}, \vec{\mu}\right)
\end{gathered}
$$

for every $\vec{\mu} \in\left(E_{2}\right)^{n}$. Then, we have $d(\Lambda(\omega), \omega)<1$.

Now, Theorem 1 enables us to find an element $\omega_{0}$ in $B$ satisfying the following:

(1) $\omega_{0}$ is a fixed point of $\Lambda$, i.e.,

$$
\begin{aligned}
\omega_{0}(p) & =\Lambda\left(\omega_{0}(p)\right) \\
& =\boldsymbol{S}\left(\sigma, \omega_{0}(\sigma)\right)+{ }_{0} \mathcal{I}_{p}^{\kappa, \psi}\left(\mathcal{M}\left(\sigma, \varepsilon, \omega_{0}(\varepsilon)\right) d \varepsilon\right),
\end{aligned}
$$

which is unique in the set:

$$
B^{*}=\{\Omega \in B: d(\Lambda(\omega), \Omega)<\infty\} .
$$

(2) $d\left(\Lambda^{k}(\omega), \omega_{0}\right) \rightarrow 0$ as $n \rightarrow \infty$;

(3) $d\left(\omega, \omega_{0}\right) \leq N_{9} d(\Lambda(\omega), \omega) \leq N_{9}$,

where $N_{9}=\frac{\min \left[L_{1}, L_{2} L_{4}\right]}{\min \left[L_{1}, L_{2} L_{4}\right]-2}$ and implying that:

$$
\Theta\left(\omega\left(p_{1}\right)-\omega_{0}\left(p_{1}\right), \cdots, \omega\left(p_{k}\right)-\omega_{0}\left(p_{k}\right), \vec{\mu}\right) \succeq \phi\left(p_{1}, \cdots, p_{k}, \frac{\vec{\mu}}{N_{9}}\right),
$$

for every $p_{1}, \cdots, p_{k} \in E_{1}$ and $\vec{\mu} \in\left(E_{2}\right)^{n}$. We use the same method of proving Theorem 3 and show that the fixed point in $B^{*}$ is unique.

\section{Examples}

In this section, we apply the main results to solve some examples.

Example 1. Let $(\mathbb{R}, \Theta, \circledast)$ be an MVF-k-B-space. Let $\Omega, Y: E_{1} \rightarrow \mathbb{R}$ such that $\Omega\left(p_{i}\right)=p_{i}{ }^{4}$ and $Y\left(p_{i}\right)=p_{i}{ }^{2}$, and define $S\left(p_{i}, \Omega\left(p_{i}\right)\right)=\frac{1}{L_{1}} \Omega\left(p_{i}\right)$. Define $\mathcal{M}: E_{1} \times E_{1} \times \mathbb{R} \rightarrow \mathbb{R}$ as $\mathcal{M}\left(p_{i}, \sigma_{i}, \Omega\left(\sigma_{i}\right)\right)=\exp \left(p_{i}{ }^{2}-\sigma_{i}{ }^{2}\right) \Omega\left(\sigma_{i}\right)$ for every $p_{i} \in E_{1}$ and $\sigma_{i} \preceq p_{i} \quad(i=1,2, \cdots, k)$.

Then, we have: 


$$
\begin{aligned}
& \Theta\left(S\left(p_{1}, \omega\left(p_{1}\right)\right)-\boldsymbol{S}\left(p_{1}, \mathrm{Y}\left(p_{1}\right)\right), \cdots, \boldsymbol{S}\left(p_{1}, \omega\left(p_{k}\right)\right)-\boldsymbol{S}\left(p_{k}, \mathrm{Y}\left(p_{k}\right)\right), \vec{\mu}\right) \\
& =\Theta\left(\frac{1}{L_{1}} \Omega\left(p_{1}\right)-\frac{1}{L_{1}} \mathrm{Y}\left(p_{1}\right), \cdots, \frac{1}{L_{1}} \Omega\left(p_{k}\right)-\frac{1}{L_{1}} \mathrm{Y}\left(p_{k}\right), \vec{\mu}\right) \\
& =\Theta\left(\frac{1}{L_{1}} p_{1}^{4}-\frac{1}{L_{1}} p_{1}^{2}, \cdots, \frac{1}{L_{1}} p_{k}^{4}-\frac{1}{L_{1}} p_{k}^{2}, \vec{\mu}\right) \\
& =\operatorname{diag}\left[\exp \left(\frac{-\frac{1}{L_{1}}\left|p_{1}^{4}-p_{1}^{2}, \cdots, p_{k}^{4}-p_{k}^{2}\right|}{\mu_{1}}\right), \frac{\mu_{2}}{\mu_{2}+\frac{1}{L_{1}}\left|p_{1}^{4}-p_{1}^{2}, \cdots, p_{k}^{4}-p_{k}^{2}\right|}, \exp \left(\frac{-\frac{1}{L_{1}}\left|p_{1}^{4}-p_{1}^{2}, \cdots, p_{k}^{4}-p_{k}^{2}\right|}{\mu_{3}}\right)\right] \\
& \succeq \operatorname{diag}\left[\exp \left(\frac{-\left|p_{1}^{4}-p_{1}^{2}, \cdots, p_{k}^{4}-p_{k}^{2}\right|}{L_{1} \mu_{1}}\right), \frac{L_{1} \mu_{2}}{L_{1} \mu_{2}+\left|p_{1}^{4}-p_{1}^{2}, \cdots, p_{k}^{4}-p_{k}^{2}\right|}, \exp \left(\frac{-\left|p_{1}^{4}-p_{1}^{2}, \cdots, p_{k}^{4}-p_{k}^{2}\right|}{L_{1} \mu_{3}}\right)\right] \\
& =\Theta\left(p_{1}^{4}-p_{1}^{2}, \cdots, p_{k}^{4}-p_{k}^{2}, L_{1} \vec{\mu}\right) \\
& =\Theta\left(\Omega\left(p_{1}\right)-\mathrm{Y}\left(p_{1}\right), \cdots, \Omega\left(p_{k}\right)-\mathrm{Y}\left(p_{k}\right), L_{1} \vec{\mu}\right), \\
& \Theta\left(\mathcal{M}\left(p_{1}, \sigma_{1}, \Omega\left(\sigma_{1}\right)\right)-\mathcal{M}\left(p_{1}, \sigma_{1}, \mathrm{Y}\left(\sigma_{1}\right)\right), \cdots, \mathcal{M}\left(p_{k}, \sigma_{k}, \Omega\left(\sigma_{k}\right)\right)-\mathcal{M}\left(p_{k}, \sigma_{k}, \mathrm{Y}\left(\sigma_{k}\right)\right), \vec{\mu}\right) \\
& =\Theta\left(\exp \left(p_{1}^{2}-\sigma_{1}{ }^{2}\right) \sigma_{1}{ }^{4}-\exp \left(p_{1}^{2}-\sigma_{1}{ }^{2}\right) \sigma_{1}{ }^{2}, \cdots, \exp \left(p_{k}^{2}-\sigma_{k}{ }^{2}\right) \sigma_{k}{ }^{4}-\exp \left(p_{k}^{2}-\sigma_{k}{ }^{2}\right) \sigma_{k}{ }^{2}, \vec{\mu}\right) \\
& =\operatorname{diag}\left[\exp \left(\frac{-\left|\exp \left(p_{1}^{2}-\sigma_{1}{ }^{2}\right) \sigma_{1}{ }^{4}-\exp \left(p_{1}^{2}-\sigma_{1}{ }^{2}\right) \sigma_{1}{ }^{2}, \ldots, \exp \left(p_{k}^{2}-\sigma_{k}{ }^{2}\right) \sigma_{k}{ }^{4}-\exp \left(p_{k}^{2}-\sigma_{k}{ }^{2}\right) \sigma_{k}{ }^{2}\right|}{\mu_{1}}\right)\right. \\
& \frac{\mu_{2}}{\mu_{2}+\left|\exp \left(p_{1}^{2}-\sigma_{1}^{2}\right) \sigma_{1}^{4}-\exp \left(p_{1}^{2}-\sigma_{1}^{2}\right) \sigma_{1}^{2}, \cdots, \exp \left(p_{k}^{2}-\sigma_{k}^{2}\right) \sigma_{k}^{4}-\exp \left(p_{k}^{2}-\sigma_{k}^{2}\right) \sigma_{k}^{2}\right|^{\prime}} \\
& \left.\exp \left(\frac{-\left|\exp \left(p_{1}^{2}-\sigma_{1}{ }^{2}\right) \sigma_{1}{ }^{4}-\exp \left(p_{1}^{2}-{\sigma_{1}}^{2}\right) \sigma_{1}{ }^{2}, \cdots, \exp \left(p_{k}^{2}-\sigma_{k}{ }^{2}\right) \sigma_{k}{ }^{4}-\exp \left(p_{k}^{2}-\sigma_{k}{ }^{2}\right) \sigma_{k}{ }^{2}\right|}{\mu_{3}}\right)\right] \\
& \succeq \operatorname{diag}\left[\exp \left(\frac{-\left|\sigma_{1}^{4}-\sigma_{1}^{2}, \cdots, \sigma_{k}^{4}-\sigma_{k}^{2}\right|}{\frac{\mu_{1}}{\exp \left(p_{1}^{2}-\sigma_{1}^{2}\right), \cdots, \exp \left(p_{k}^{2}-\sigma_{k}^{2}\right)}}\right), \frac{\frac{\mu_{2}}{\exp \left(p_{1}^{2}-\sigma_{1}^{2}\right), \cdots, \exp \left(p_{k}^{2}-\sigma_{k}^{2}\right)}}{\frac{\mu_{2}}{\exp \left(p_{1}^{2}-\sigma_{1}^{2}\right), \cdots, \exp \left(p_{k}^{2}-\sigma_{k}^{2}\right)}+\left|\sigma_{1}^{4}-\sigma_{1}^{2}, \cdots, \sigma_{k}^{4}-\sigma_{k}^{2}\right|},\right. \\
& \left.\exp \left(\frac{-\left|\sigma_{1}^{4}-\sigma_{1}^{2}, \cdots, \sigma_{k}^{4}-\sigma_{k}^{2}\right|}{\frac{\mu_{3}}{\exp \left(p_{1}^{2}-\sigma_{1}^{2}\right), \cdots, \exp \left(p_{k}^{2}-\sigma_{k}^{2}\right)}}\right)\right] \\
& =\Theta\left(\Omega\left(\sigma_{1}\right)-\mathrm{Y}\left(\sigma_{1}\right), \cdots, \Omega\left(\sigma_{k}\right)-\mathrm{Y}\left(\sigma_{k}\right), \frac{\vec{\mu}}{\left|\exp \left(p_{1}^{2}-\sigma_{1}^{2}\right), \cdots, \exp \left(p_{k}^{2}-\sigma_{k}^{2}\right)\right|}\right) \\
& \succeq \Theta\left(\Omega\left(\sigma_{1}\right)-\mathrm{Y}\left(\sigma_{1}\right), \cdots, \Omega\left(\sigma_{k}\right)-\mathrm{Y}\left(\sigma_{k}\right), \frac{\vec{\mu}}{K}\right)
\end{aligned}
$$

for some $K \in E_{6}$.

Let $\operatorname{MVFF} \phi:\left(E_{1}\right)^{k} \times\left(E_{2}\right)^{3} \rightarrow \operatorname{diag} M_{n}\left(E_{3}\right)$, satisfying (6) and (7).

Let $\omega: E_{1} \rightarrow \mathbb{R}$ be a differentiable function satisfying:

$$
\begin{aligned}
& \operatorname{diag}\left[\exp \left(\frac{-{ }_{0} \mathcal{D}_{p_{1}}^{\kappa, \tau, \psi} \omega\left(p_{1}\right)-\frac{1}{L_{1}} \omega\left(p_{1}\right)-\int_{0}^{p_{1}} \exp \left(p_{1}^{2}-\sigma_{1}{ }^{2}\right) \omega\left(\sigma_{1}\right) d \sigma_{1}, \cdots,{ }_{0} \mathcal{D}_{p_{k}}^{\kappa, \tau, \psi} \omega\left(p_{k}\right)-\frac{1}{L_{1}} \omega\left(p_{k}\right)-\int_{0}^{p_{k}} \exp \left(p_{k}^{2}-\sigma_{k}{ }^{2}\right) \omega\left(\sigma_{k}\right) d \sigma_{k} \mid}{\mu_{1}}\right),\right. \\
& \frac{\mu_{2}}{\mu_{2}+{ }_{0} \mathcal{D}_{p_{1}}^{\kappa, \tau, \psi} \omega\left(p_{1}\right)-\frac{1}{L_{1}} \omega\left(p_{1}\right)-\int_{0}^{p_{1}} \exp \left(p_{1}^{2}-\sigma_{1}^{2}\right) \omega\left(\sigma_{1}\right) d \sigma_{1}, \cdots,{ }_{0} \mathcal{D}_{p_{k}}^{\kappa, \tau, \psi} \omega\left(p_{k}\right)-\frac{1}{L_{1}} \omega\left(p_{k}\right)-\left.\int_{0}^{p_{k}} \exp \left(p_{k}^{2}-\sigma_{k}{ }^{2}\right) \omega\left(\sigma_{k}\right) d \sigma_{k}\right|^{2}}, \\
& \left.\exp \left(\frac{-\left.\right|_{0} \mathcal{D}_{p_{1}}^{\kappa, \tau, \psi} \omega\left(p_{1}\right)-\frac{1}{L_{1}} \omega\left(p_{1}\right)-\int_{0}^{p_{1}} \exp \left(p_{1}^{2}-\sigma_{1}^{2}\right) \omega\left(\sigma_{1}\right) d \sigma_{1}, \cdots, 0}{\mathcal{D}_{p_{k}}^{\kappa, \tau, \psi} \omega\left(p_{k}\right)-\frac{1}{L_{1}} \omega\left(p_{k}\right)-\int_{0}^{p_{k}} \exp \left(p_{k}^{2}-\sigma_{k}{ }^{2}\right) \omega\left(\sigma_{k}\right) d \sigma_{k} \mid}\right)\right] \\
& \succeq \operatorname{diag}\left[\exp \left(\frac{-\left|p_{1}, \cdots, p_{k}\right|}{\mu_{1}}\right), \frac{\mu_{2}}{\mu_{2}+\left|p_{1}, \cdots, p_{k}\right|}, \exp \left(\frac{-\left|p_{1}, \cdots, p_{k}\right|}{\mu_{3}}\right)\right] \text {. }
\end{aligned}
$$

for every $p_{1}, \cdots, p_{k} \in E_{1}$ and $\vec{\mu} \in\left(E_{2}\right)^{3}$. Now, Theorem 3 implies that, if $H_{1}>2, H_{1}=$ 
$\min \left[L_{1}, \frac{L_{3}}{K}, L_{1} L_{4}, \frac{L_{3} L_{4}}{K}\right]$. We are to be able find a unique differentiable function $\omega_{0}: E_{1} \rightarrow \mathbb{R}$ such that:

$$
{ }_{0} \mathcal{D}_{p}^{\kappa, \tau, \psi} \omega_{0}(p)=\frac{1}{L_{1}} \omega_{0}(p)+\int_{0}^{p} \exp \left(p^{2}-\sigma^{2}\right) \omega(\sigma) d \sigma
$$

and:

$$
\begin{aligned}
& \operatorname{diag}\left[\exp \left(\frac{-\left.\right|_{0} \mathcal{D}_{p_{1}}^{\kappa, \tau, \psi} \omega\left(p_{1}\right)-{ }_{0} \mathcal{D}_{p_{1}}^{\kappa, \tau, \psi} \omega_{0}\left(p_{1}\right), \cdots{ }_{0} \mathcal{D}_{p_{k}}^{\kappa, \tau, \psi} \omega\left(p_{k}\right)-{ }_{0} \mathcal{D}_{p_{k}}^{\kappa, \tau, \psi} \omega_{0}\left(p_{k}\right) \mid}{\mu_{1}}\right),\right. \\
& \frac{\mu_{2}}{\mu_{2}+\left|{ }_{0} \mathcal{D}_{p_{1}}^{\kappa, \tau, \psi} \omega\left(p_{1}\right)-{ }_{0} \mathcal{D}_{p_{1}}^{\kappa, \tau, \psi} \omega_{0}\left(p_{1}\right), \cdots{ }_{0} \mathcal{D}_{p_{k}}^{\kappa, \tau, \psi} \omega\left(p_{k}\right)-{ }_{0} \mathcal{D}_{p_{k}}^{\kappa, \tau, \psi} \omega_{0}\left(p_{k}\right)\right|}, \\
& \left.\exp \left(\frac{-\left|{ }_{0} \mathcal{D}_{p_{1}}^{\kappa, \tau, \psi} \omega\left(p_{1}\right)-{ }_{0} \mathcal{D}_{p_{1}}^{\kappa, \tau, \psi} \omega_{0}\left(p_{1}\right), \cdots{ }_{0} \mathcal{D}_{p_{k}}^{\kappa, \tau, \psi} \omega\left(p_{k}\right)-{ }_{0} \mathcal{D}_{p_{k}}^{\kappa, \tau, \psi} \omega_{0}\left(p_{k}\right)\right|}{\mu_{3}}\right)\right] \\
& \circledast \operatorname{diag}\left[\exp \left(\frac{-\left|\omega\left(p_{1}\right)-\omega_{0}\left(p_{1}\right), \cdots, \omega\left(p_{k}\right)-\omega_{0}\left(p_{k}\right)\right|}{\mu_{1}}\right), \frac{\mu_{2}}{\mu_{2}+\left|\omega\left(p_{1}\right)-\omega_{0}\left(p_{1}\right), \cdots, \omega\left(p_{k}\right)-\omega_{0}\left(p_{k}\right)\right|^{\prime}}\right. \text {, } \\
& \left.\exp \left(\frac{-\left|\omega\left(p_{1}\right)-\omega_{0}\left(p_{1}\right), \cdots, \omega\left(p_{k}\right)-\omega_{0}\left(p_{k}\right)\right|}{\mu_{3}}\right)\right] \\
& \succeq \operatorname{diag}\left[\exp \left(\frac{\left(-\left|p_{1}, \cdots, p_{k}\right|\right)}{\frac{N_{1}}{H_{2}} \mu_{1}}\right), \frac{\frac{N_{1}}{H_{2}} \mu_{2}}{\frac{N_{1}}{H_{2}} \mu_{2}+\left|p_{1}, \cdots, p_{k}\right|},\right. \\
& \left.\exp \left(\frac{\left(-\left|p_{1}, \cdots, p_{k}\right|\right)}{\frac{N_{1}}{H_{2}} \mu_{3}}\right)\right] \text {, }
\end{aligned}
$$

for every $p_{1}, \cdots, p_{k} \in E_{1}$ and $\vec{\mu} \in\left(E_{2}\right)^{3}$, and $N_{1}=\min \left[1, L_{4}\right]$ and $H_{2}=\frac{\min \left[L_{1}, \frac{L_{3}}{K}, L_{1} L_{4}, \frac{L_{3} L_{4}}{K}\right]}{\min \left[L_{1}, \frac{L_{3}}{K}, L_{1} L_{4}, \frac{L_{3} L_{4}}{K}\right]-2}$.

Example 2. Let $(\mathbb{R}, \Theta, \circledast)$ be an MVF-k-B-space. Consider $\Omega, Y: E_{1} \rightarrow \mathbb{R}$ such that $\Omega\left(p_{i}\right)=$ $p_{i}{ }^{4}$ and $\mathrm{Y}\left(p_{i}\right)=p_{i}{ }^{2}$, and define $S\left(p_{i}, \Omega\left(p_{i}\right)\right)=\frac{1}{L_{1}} \Omega\left(p_{i}\right)$. Define $\mathcal{M}: E_{1} \times E_{1} \times \mathbb{R} \rightarrow \mathbb{R}$ as $\mathcal{M}\left(p_{i}, \sigma_{i}, \Omega\left(\sigma_{i}\right)\right)=\exp \left(p_{i}{ }^{2}-\sigma_{i}{ }^{2}\right) \Omega\left(\sigma_{i}\right)$ for every $p_{1}, \cdots, p_{k} \in E_{1}$ and $\sigma_{i} \preceq p_{i} \quad(i=$ $1,2, \cdots, k)$ satisfying $(49)$.

Let MVFF $\phi:\left(E_{1}\right)^{k} \times\left(E_{2}\right)^{3} \rightarrow \operatorname{diag} M_{n}\left(E_{3}\right)$, satisfying (6) and (7).

Let $\omega: E_{1} \rightarrow \mathbb{R}$ be a differentiable function satisfying:

$$
\begin{array}{r}
\operatorname{diag}\left[\exp \left(\frac{-\left|\omega\left(p_{1}\right)-\frac{1}{L_{1}} \omega\left(p_{1}\right)-{ }_{0} \mathcal{I}_{p_{1}}^{\kappa, \psi} \exp \left(p_{1}^{2}-\sigma_{1}^{2}\right) \omega\left(\sigma_{1}\right) d \sigma_{1}, \cdots, \omega\left(p_{k}\right)-\frac{1}{L_{1}} \omega\left(p_{k}\right)-{ }_{0} \mathcal{I}_{p_{k}}^{\kappa, \psi} \exp \left(p_{k}^{2}-\sigma_{k}{ }^{2}\right) \omega\left(\sigma_{k}\right) d \sigma_{k}\right|}{\mu_{1}}\right)\right. \\
, \frac{\mu_{2}}{\mu_{2}+\left|\omega\left(p_{1}\right)-\frac{1}{L_{1}} \omega\left(p_{1}\right)-{ }_{0} \mathcal{I}_{p_{1}}^{\kappa, \psi} \exp \left(p_{1}^{2}-\sigma_{1}{ }^{2}\right) \omega\left(\sigma_{1}\right) d \sigma_{1}, \cdots, \omega\left(p_{k}\right)-\frac{1}{L_{1}} \omega\left(p_{k}\right)-{ }_{0} \mathcal{I}_{p_{k}}^{\kappa, \psi} \exp \left(p_{k}^{2}-\sigma_{k}{ }^{2}\right) \omega\left(\sigma_{k}\right) d \sigma_{k}\right|} \\
\left., \exp \left(\frac{-\left|\omega\left(p_{1}\right)-\frac{1}{L_{1}} \omega\left(p_{1}\right)-{ }_{0} \mathcal{I}_{p_{1}}^{\kappa, \psi} \exp \left(p_{1}^{2}-\sigma_{1}{ }^{2}\right) \omega\left(\sigma_{1}\right) d \sigma_{1}, \cdots, \omega\left(p_{k}\right)-\frac{1}{L_{1}} \omega\left(p_{k}\right)-{ }_{0} \mathcal{I}_{p_{k}}^{\kappa, \psi} \exp \left(p_{k}^{2}-\sigma_{k}{ }^{2}\right) \omega\left(\sigma_{k}\right) d \sigma_{k}\right|}{\mu_{3}}\right)\right] \\
\succeq \operatorname{diag}\left[\exp \left(\frac{-\left|p_{1}, \cdots, p_{k}\right|}{\mu_{1}}\right), \frac{\mu_{2}}{\mu_{2}+\left|p_{1}, \cdots, p_{k}\right|}, \exp \left(\frac{-\left|p_{1}, \cdots, p_{k}\right|}{\mu_{3}}\right)\right]
\end{array}
$$

for every $p_{1}, \cdots, p_{k} \in E_{1}$ and $\vec{\mu} \in\left(E_{2}\right)^{3}$.

Now, Theorem 4 implies that, if $H_{3}>2, H_{3}=\min \left[L_{1}, \frac{L_{4}}{K}\right]$. We are to be able find a unique differentiable function $\omega_{0}: E_{1} \rightarrow \mathbb{R}$ such that:

$$
\omega_{0}(p)=\frac{1}{L_{1}} \omega_{0}(p)+{ }_{0} \mathcal{I}_{p}^{\kappa, \psi} \exp \left(p^{2}-\sigma^{2}\right) \omega(\sigma) d \sigma,
$$


and:

$$
\begin{gathered}
\operatorname{diag}\left[\exp \left(\frac{-\left|\omega\left(p_{1}\right)-\omega_{0}\left(p_{1}\right), \cdots, \omega\left(p_{k}\right)-\omega_{0}\left(p_{k}\right)\right|}{\mu_{1}}\right), \frac{\mu_{2}}{\mu_{2}+\left|\omega\left(p_{1}\right)-\omega_{0}\left(p_{1}\right), \cdots, \omega\left(p_{k}\right)-\omega_{0}\left(p_{k}\right)\right|},\right. \\
\left.\quad \exp \left(\frac{-\left|\omega\left(p_{1}\right)-\omega_{0}\left(p_{1}\right), \cdots, \omega\left(p_{k}\right)-\omega_{0}\left(p_{k}\right)\right|}{\mu_{3}}\right)\right] \\
\succeq \operatorname{diag}\left[\exp \left(\frac{-\left|p_{1}, \cdots, p_{k}\right|}{\frac{N_{1}}{H_{4}} \mu_{1}}\right), \frac{\frac{N_{1}}{H_{4}} \mu_{2}}{\frac{N_{1}}{H_{4}} \mu_{2}+\left|p_{1}, \cdots, p_{k}\right|}, \exp \left(\frac{-\left|p_{1}, \cdots, p_{k}\right|}{\frac{N_{1}}{H_{4}} \mu_{3}}\right)\right]
\end{gathered}
$$

for every $p_{1}, \cdots, p_{k} \in E_{1}$ and $\vec{\mu} \in\left(E_{2}\right)^{3}$, and $N_{2}=\min \left[1, L_{4}\right]$ and $H_{4}=\frac{\min \left[L_{1}, \frac{L_{4}}{K}\right]}{\min \left[L_{1}, \frac{L_{4}}{K}\right]-2}$.

\section{Conclusions}

In this paper, we studied the concept of matrix-valued fuzzy $k$-normed spaces (MVF$\mathrm{k}-\mathrm{N}$-spaces), and we applied the alternative fixed-point theorem to investigate the HyersUlam-Rassias stability of some fractional equations in these spaces. We defined a class of matrix-valued fuzzy control functions for stabilizing fractional Volterra integro-differential equations with $\psi$-Hilfer fractional derivative in the complete MVF-k-N-spaces, and we obtained the best approximation for this kind of fractional equations.

Author Contributions: All authors conceived of the study, participated in its design and coordination, drafted the manuscript, and participated in the sequence alignment. All authors read and agreed to the published version of the manuscript.

Funding: The authors are grateful to the Basque Government by the support of this work through Grant IT1207-19.

Institutional Review Board Statement: Not applicable.

Informed Consent Statement: Not applicable.

Data Availability Statement: Not applicable.

Acknowledgments: The authors are thankful to the Area Editor and the referees for giving valuable comments and suggestions.

Conflicts of Interest: The authors declare no conflict of interest.

\section{References}

1. Almeida, R.; Tavares, D.; Torres, D.F.M. The Variable-Order Fractional Calculus of Variations; Springer Briefs in Applied Sciences and Technology; Springer: Cham, Switzerland, 2019.

2. Muniyappan, P.; Rajan, S. Stability of a class of fractional integro-differential equation with nonlocal initial conditions. Acta Math. Univ. Comenian. 2018, 87, 85-95.

3. Selvam, A.G.M.; Baleanu, D.; Alzabut, J.; Vignesh, D.; Abbas, S. On Hyers-Ulam Mittag-Leffler stability of discrete fractional Duffing equation with application on in- verted pendulum. Adv. Differ. Equ. 2020, 456, 1-15.

4. Agarwal, R.P.; Hristova, S.; O'Regan, D. Lyapunov functions to Caputo reaction-diffusion fractional neural networks with time-varying delays. J. Math. Comput. Sci. 2018, 18, 328-345. [CrossRef]

5. Da Sousa, J.V.C.; Fabio, G.R.; de Oliveira, E.C. Stability of the fractional Volterra integro-differential equation by means of $\Psi$-Hilfer operator. Math. Meth. Appl. Sci. 2019, 42, 3033-3043. [CrossRef]

6. Hadzic, O.; Pap, E. Fixed Point Theory in Probabilistic Metric Spaces. In Mathematics and its Applications; Kluwer Academic Publishers: Dordrecht, The Netherlands, 2001.

7. Schweizer, B.; Sklar, A. North-Holland Series in Probability and Applied Mathematics; North-Holland Publishing Co.: New York, NY, USA, 1983.

8. Klement, E.P.; Mesiar, R.; Pap, E. Triangular norms. In Trends in Logic-Studia Logica Library; Kluwer Academic Publishers: Dordrecht, The Netherlands, 2000.

9. Cădariu, L.; Radu, V. Fixed points and the stability of Jensen's functional equation. JIPAM J. Inequal. Pure Appl. Math. $2003,4,7$.

10. Diaz, J.B.; Margolis, B. A fixed point theorem of the alternative, for contractions on a generalized complete metric space. Bull. Amer. Math. Soc. 1968, 74, 305-309. [CrossRef]

11. Chaharpashlou, R.; Saadati, R.; Atangana, A. Ulam-Hyers-Rassias stability for nonlinear $\Psi$-Hilfer stochastic fractional differential equation with uncertainty. Adv. Differ. Equ. 2020, 339, 10. [CrossRef] 
12. El-Sayed, A.M.A.; Al-Issa, S.M. Existence of integrable solutions for integro-differential inclusions of fractional order; coupled system approach. J. Nonlinear Sci. Appl. 2020, 13, 180-186. [CrossRef]

13. Khan O.; Araci S.; Saif, M. Fractional calculus formulas for Mathieu-type series and generalized Mittag-Leffler function. J. Math Comput. SCI-JM 2020, 20, 122-130. [CrossRef]

14. Sene, N. Global asymptotic stability of the fractional differential equations. J. Nonlinear Sci. Appl. 2020, 13, 171-175. [CrossRef]

15. Cădariu, L.; Radu, V. On the stability of the Cauchy functional equation: A fixed point approach. Grazer Math. Ber. 2004, 346, 43-52.

16. Miheț, D.; Radu, V. On the stability of the additive Cauchy functional equation in random normed spaces. J. Math. Anal. Appl. 2008, 343, 567-572. [CrossRef]

17. Alzabut, J.; Ahmad, B.; Etemad, S.; Rezapour, S.; Zada, A. Novel existence techniques on the generalized $\phi$-Caputo fractional inclusion boundary problem. Adv. Differ. Equ. 2021, 135, 1-18.

18. Matar, M.M.; Abbas, M.I.; Alzabut, J.; Kaabar, M.K.A.; Etemad, S.; Rezapour, S. Investigation of the $p$-Laplacian nonperiodic nonlinear boundary value problem via generalized Caputo fractional derivatives. Adv. Differ. Equ. 2021, 68, 18.

19. Zhou, H.; Alzabut, J.; Rezapour, S.; Samei, M.E. Uniform persistence and almost periodic solutions of a nonautonomous patch occupancy model. Adv. Differ. Equ. 2020, 143, 12. [CrossRef]

20. Rezapour, S.; Imran, A.; Hussain, A.; Martinez, F.; Etemad, S.; Kaabar, M.K.A. Condensing Functions and Approximate Endpoint Criterion for the Existence Analysis of Quantum Integro-Difference FBVPs. Symmetry 2021, 13, 469. [CrossRef]

21. Korovina, M.V.; Matevossian, H.; Smirnov, I.N. On the Asymptotics of Solutions of the Wave Operator with Meromorphic Coefficients. Mat. Zametki 2021, 109, 312-317.

22. Matevossian, H.A. On the biharmonic problem with the Steklov-type and farwig boundary conditions. Lobachevskii J. Math. 2020, 41, 2053-2059. [CrossRef]

23. Al-Issa, S.M.; Mawed, N.M. Results on solvability of nonlinear quadratic integral equations of fractional orders in Banach algebra. J. Nonlinear Sci. Appl. 2021, 14, 181-195. [CrossRef]

24. Satsanit, W. On the solution linear and nonlinear fractional beam equation. J. Nonlinear Sci. Appl. 2021, 14, 139-147. [CrossRef]

25. Rezaei Aderyani, S.; Saadati, R. Best approximations of the $\varphi$-Hadamard fractional Volterra integro-differential equation by matrix-valued fuzzy control functions. Adv. Differ. Equ. 2021, 154, 1-21.

26. Chaharpashlou, R.; Saadati, R. Best approximation of a nonlinear fractional Volterra integro-differential equation in matrix MB-space. Adv. Differ. Equ. 2021, 118, 1-12.

27. Asaduzzamana, M.; Kilicmanb, A.; Alic, M.Z. Presence and diversity of positive solutions for a Caputo-type fractional order nonlinear differential equation with an advanced argument. J. Math. Comput. Sci. 2021, 23, 230-244. [CrossRef]

28. Ababneh $\mathrm{O}$. Adaptive synchronization and anti-synchronization of fractional order chaotic optical systems with uncertain parameters. J. Math. Comput. Sci. 2021, 23, 302-314. [CrossRef]

29. El-Moneam, M.A.; Ibrahim, T.F.; Elamody, S. Stability of a fractional difference equation of high order. J. Nonlinear Sci. Appl. 2019, 12, 65-74. [CrossRef]

30. Golet, I. Some remarks on functions with values in probabilistic normed spaces. Math. Slovaca 2007, 57, 259-270. [CrossRef]

31. Saadati, R. Nonlinear contraction and fuzzy compact operator in fuzzy Banach algebras. Fixed Point Theory 2019, $20,289-297$. [CrossRef]

32. S̆erstnev, A.N. On the notion of a random normed space. Dokl. Akad. Nauk SSSR 1963, 149, 280-283. (In Russian)

33. Sousa, J.; da Vanterler, C.; de Oliveira, E. Capelas. On a new operator in fractional calculus and applications. J. Fixed Point Theory Appl. 2018, 20, 21.

34. Wang, J.R.; Zhou, Y. Mittag-Leffler-Ulam stabilities of fractional evolution equations. Appl. Math. Lett. 2012, 25, 723-728. [CrossRef] 